\title{
Severe asthma in humans and mouse model suggests a CXCL10 signature underlies corticosteroid-resistant Th1 bias
}

\author{
Marc Gauthier, ${ }^{1}$ Krishnendu Chakraborty, ${ }^{1}$ Timothy B. Oriss, ${ }^{1}$ Mahesh Raundhal, ${ }^{1}$ Sudipta Das, ${ }^{1}$ \\ Jie Chen, ${ }^{1}$ Rachael Huff, ${ }^{1}$ Ayan Sinha, ${ }^{1}$ Merritt Fajt, ${ }^{1,2}$ Prabir Ray, ${ }^{1,2,3}$ Sally E. Wenzel, ${ }^{1,2,3}$ \\ and Anuradha Ray ${ }^{1,2,3}$ \\ 'Division of Pulmonary, Allergy, and Critical Care Medicine, Department of Medicine, ${ }^{2}$ University of Pittsburgh Asthma \\ Institute at University of Pittsburgh Medical Center (UPMC), and 'Department of Immunology, University of Pittsburgh \\ School of Medicine, Pittsburgh, Pennsylvania, USA.
}

\begin{abstract}
We previously showed that Th1/type 1 inflammation marked by increased IFN- $\gamma$ levels in the airways can be appreciated in $\mathbf{5 0} \%$ of patients with severe asthma, despite high dose corticosteroid (CS) treatment. We hypothesized that a downstream target of IFN- $\gamma$, CXCL10, which recruits Th1 cells via the cognate receptor CXCR3, is an important contributor to Th1 1igh asthma and CS unresponsiveness. We show high levels of CXCL10 mRNA closely associated with IFNG levels in the BAL cells of $50 \%$ of severe asthmatics and also in the airways of mice subjected to a severe asthma model, both in the context of high-dose CS treatment. The inability of CS to dampen IFNG or CXCL10 expression was not because of impaired nuclear translocation of the glucocorticoid receptor (GR) or its transactivational functions. Rather, in the presence of CS and IFN- $\gamma$, STAT1 and GR were recruited on critical regulatory elements in the endogenous CXCL10 promoter in monocytes, albeit without any abatement of CXCL10 gene expression. High CXCL10 gene expression was also associated with a mast cell signature in both humans and mice, CXCR3 being also expressed by mast cells. These findings suggest that the IFN- $\gamma$-CXCL10 axis plays a central role in persistent type 1 inflammation that may be facilitated by CS therapy through GR-STAT1 cooperation converging on the CXCL10 promoter.
\end{abstract}

Conflict of interest: The authors have declared that no conflict of interest exists.

Submitted: April 13, 2017

Accepted: May 25, 2017

Published: July 6, 2017

Reference information: JCI Insight. 2017;2(13):e94580. https://doi.org/10.1172/jci. insight. 94580 .

\section{Introduction}

Asthma is a disease with significant prevalence and morbidity throughout the developed world, affecting nearly $5 \%-10 \%$ of populations (1). It is increasingly recognized that asthma is a disease with multiple phenotypes, each with its own unique molecular mechanisms, natural history, and response to therapy $(2,3)$. Corticosteroids (CS) have remained the mainstay of therapy, but for many patients, these therapies are ineffective (4), and research of underlying mechanisms may give insight into the etiologies of CS resistance and identify future therapeutic targets.

Our previous studies have shown that the number of Th1 cells, which produce IFN- $\gamma$, is elevated in approximately $50 \%$ of severe asthma (SA) patients and in our mouse model of SA (5), and increased IFNG mRNA levels are also evident in the airways of these subjects (6). Furthermore, IFN- $\gamma$ was associated with increased airway hyperreactivity (AHR) and poor CS response (5). A high Th1/IFN- $\gamma$ response in any tissue is typically induced during infections by bacteria and viruses (7). Infections by viruses (rhinovirus being the most common) and bacteria have been observed in patients with SA and can trigger asthma exacerbations (7). Several bacterial species have also been associated with severe disease (8). Once generated in lung-draining lymph nodes, Th1 cells need to be recruited to the site of infection, and the best known chemoattractant for Th1 cells is CXCL10 (9), initially cloned as an IFN- $\gamma$-induced molecule from monocytes (10). Additional chemokines that belong to the same family induced by IFN- $\gamma$ include CXCL9 and CXCL11, although CXCL10 is the most studied (11). While there is significant redundancy in their effects, the expression of the 3 family members is not uniform in disease settings, including allergic disease $(11,12)$.

In this study, we sought to better understand the possible etiologies of this resistance to CS therapy, 
Table 1. Patient characteristics

\begin{tabular}{|c|c|c|c|c|}
\hline & Healthy control & Mild to moderate asthma & Severe asthma & $P$ value \\
\hline$n$ & 12 & 18 & 17 & \\
\hline European Descent & $10(0.83)^{A}$ & $10(0.55)^{A}$ & $13(0.76)^{\mathrm{A}}$ & \multirow{2}{*}{$0.50^{c}$} \\
\hline Other & $1(0.08)^{A}$ & $3(0.17)^{A}$ & $2(0.12)^{A}$ & \\
\hline Sex (\%Female) & $6(0.50)^{A}$ & $14(0.78)^{A}$ & $14(0.82)^{A}$ & $0.21^{\mathrm{C}}$ \\
\hline Age at enrollment (years) & $27.87(21.65-27.50)^{\mathrm{B}}$ & $32.59(21.16-43.76)^{\mathrm{B}}$ & $46.91(36.91-59.44)^{\mathrm{B}}$ & $0.0007^{0}$ \\
\hline AQLQ Score & $6.98(6.98-7.00)^{\mathrm{B}}$ & $4.97(4.20-5.88)^{\mathrm{B}}$ & $4.35(3.73-5.20)^{\mathrm{B}}$ & $<0.0001^{D}$ \\
\hline Atopy (\% atopic) & $5(0.42)^{A}$ & $14(0.93)^{A}$ & $11(0.79)^{A}$ & $0.009^{c}$ \\
\hline Blood eosinophils (cells/dl) & $192(100-175)^{\mathrm{B}}$ & $244(100-300)^{\mathrm{B}}$ & $306(100-400)^{\mathrm{B}}$ & $0.24^{\mathrm{D}}$ \\
\hline Blood IgE level (KU/I) & $25.50(7.75-28.75)^{\mathrm{B}}$ & $277.10(66.00-471.00)^{\mathrm{B}}$ & $181.40(27.00-260.00)^{\mathrm{B}}$ & $0.0003^{D}$ \\
\hline Fraction exhaled NO (ppb) & $25.72(17.13-39.55)^{\mathrm{B}}$ & $28.84(16.10-35.00)^{\mathrm{B}}$ & $50.34(31.00-71.05)^{\mathrm{B}}$ & $0.01^{D}$ \\
\hline$\%$ Response in FEV after albuterol & $6.13(3.97-9.18)^{\mathrm{B}}$ & $12.28(5.39-20.84)^{\mathrm{B}}$ & $21.50(8.86-32.95)^{\mathrm{B}}$ & $0.02^{D}$ \\
\hline On inhaled corticosteroids & $0(0.00)^{A}$ & $13(0.72)^{\mathrm{A}}$ & $17(1.00)^{A}$ & $<0.0001$ \\
\hline
\end{tabular}

${ }^{A}$ Number (percent of total); ${ }^{B}$ mean (25\%-75\%); ${ }^{C} \chi^{2}$ test; ${ }^{D}$ Kruskal-Wallis with Dunn's posthoc test; ${ }^{E}$ Mann-Whitney $U$ test. AQLQ, asthma quality of life questionnaires.

and as such, we focused on CXCL10, given its role in recruiting Th1 cells to reinforce type 1 inflammation to combat and eliminate viral and bacterial pathogens, a function that when uncontrolled can lead to significant pathology (13). The expression of CXCL10 can be induced not only by IFN- $\gamma$, but also by additional stimuli, including LPS, which can lead to differential levels of CXCL10 production and response to therapies $(11,14-16)$. In addition to its expression on Th1 cells, the CXCR3 receptor which mediates chemoattraction by CXCL10 and its family members - is also present on mast cells (17), neutrophils (18), and eosinophils (19). Elevated CXCL10 levels have been detected in multiple compartments, including blood and bronchoalveolar lavage (BAL) in mild and atopic asthma (20-22) and may be increased during asthma exacerbations (23). CXCL10 can be secreted by multiple cell types, including airway epithelial cells, smooth muscle cells, monocytes, and macrophages (11). However, several studies have implicated monocytes/macrophages as possible drivers of CXCL10 expression in asthma $(24,25)$.

Given its association with IFN- $\gamma$, we asked whether CXCL10 may be a contributor to steroid resistance in SA. Here, we show high levels of CXCL10 mRNA closely associated with IFNG levels in the airways of $50 \%$ of SA subjects and in mice subjected to our SA model, both in the context of high-dose CS treatment. Our investigation of possible impairment of glucocorticoid receptor (GR) function in the presence of IFN- $\gamma$ showed no such impairment with preservation of nuclear translocation and transactivational functions of GR. However, as revealed using ChIP assay, in the presence of CS and IFN- $\gamma$, we observed simultaneous enrichment of STAT1 and GR on critical regulatory elements in the endogenous CXCL10 promoter in monocytes; importantly, this did not cause inhibition of CXCL10 expression at either mRNA or protein levels. In contrast, CS inhibited LPS-induced binding of NF- $\kappa B$ to the CXCL10 promoter and inhibited LPS-induced CXCL10 gene expression, showing selective impairment of CS-mediated suppression in the presence of IFN- $\gamma$. High CXCL10 gene expression was also associated with markers of mast cells in the airways of severe asthmatics, consistent with known human mast cell expression of CXCR3. Taken together, these findings suggest that the IFN- $\gamma /$ CXCL10 axis is prominent in CS-refractory disease. Increased expression of both IFN- $\gamma$ and the chemokine that recruits IFN- $\gamma$ producing Th1 cells may establish a persistent type 1 inflammation that may actually worsen with CS therapy through GR-STAT1 cooperation in promoting CXCL10 gene expression. 


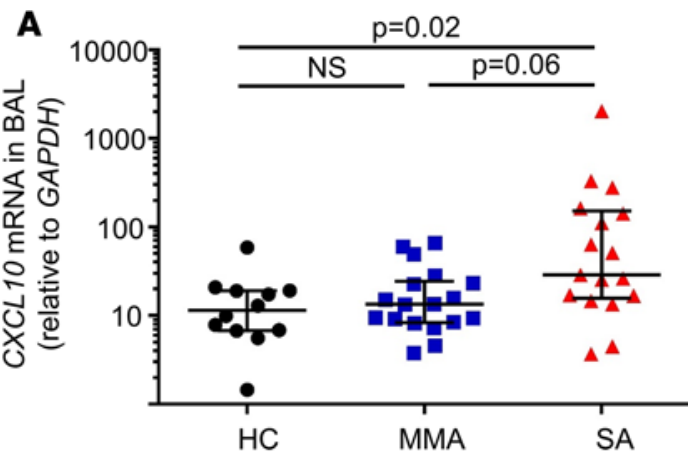

C

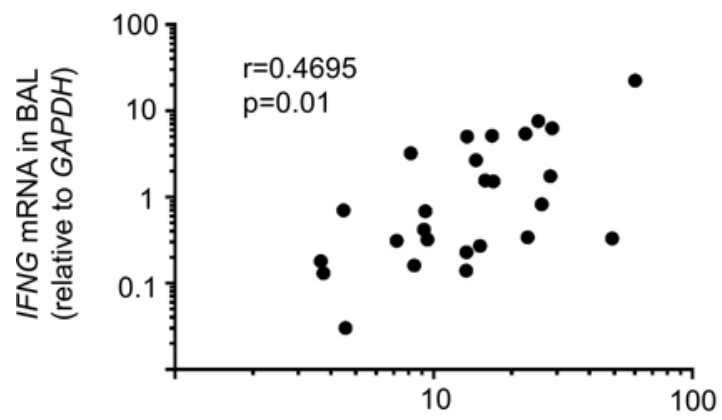

CXCL10 mRNA in BAL (relative to GAPDH)
B
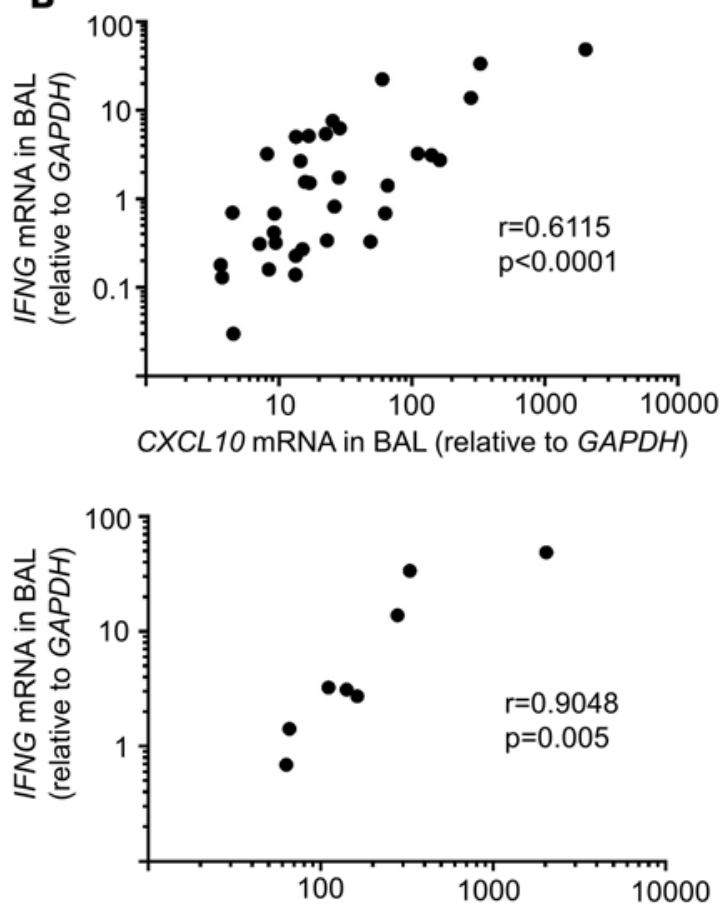

CXCL10 mRNA in BAL (relative to GAPDH)
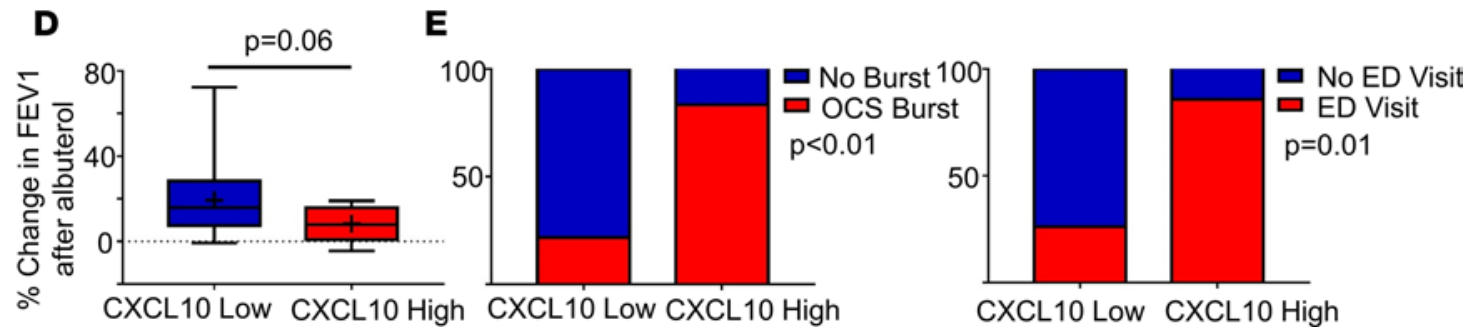

Figure 1. CXCL10 expression is elevated in severe asthma and associated with poor asthma control. (A) CXCL10 mRNA expression was measured in BAL cells obtained from patients with mild-moderate asthma (MMA, $n=18$ ), severe asthma (SA, $n=17$ ), and healthy controls (HC, $n=12$ ). (B) CXCL10 mRNA levels in BAL cells were compared with IFNG mRNA levels in all patients with asthma $(n=35)$. (C) A cut-off for CXCL10 was set at 3 SDs above the healthy control mean (CXCL10 Low $n=27$, CXCL10 High $n=8$ [all SA patients]); CXCL10 mRNA levels in BAL cells were compared with IFNG mRNA levels in each group. (D) FEV 1 was compared before and after 6 puffs of albuterol to obtain a percent change after albuterol administration. (E) Patients were asked about need for oral corticosteroid burst (OCS burst; CXCL10 Low, $n=27$; CXCL10 High, $n=7$ ) and emergency department visit (ED Visit; CXCL10 Low, $n=26$; CXCL10 High, $n=6)$ in the prior 12 months; data shown as percent of total. Error bars represent median and quartile range (A) or range with quartile range and median, \pm mean (C). Kruskal-Wallis with Dunn's posthoc test for multiple comparisons (A), Spearman's correlation (B and $\mathbf{C})$, Mann-Whitney $U$ test (D) and Fisher's Exact Test (E) were used.

\section{Results}

CXCL10 $\mathrm{mRNA}$ levels are increased in $\sim 50 \%$ of SA patients and associated with poor asthma control. CXCL10 mRNA levels were estimated in BAL cells from patients with mild to moderate $(n=18)$ or SA $(n=17)$ and from healthy controls $(n=12)$ (Table 1). Expression of CXCL10 mRNA was higher in the BAL cells of patients with SA compared with that in healthy controls (mean CXCL10 expression of 195.50 [ \pm 117.40$]$ and 15.52 [ \pm 4.32 ], respectively, $P=0.02$ ). This increased level was strongly driven by a subgroup of approximately $50 \%$ of SA patients with markedly elevated CXCL10 mRNA levels. There was no significant difference in expression between healthy control and mild to moderate asthma (mean CXCL10 expression 20.38 [ \pm 4.45$]$ in mild to moderate asthma, $P>0.99$ ), although a trend toward significance between mild to moderate and SA was noted $(P=0.06)$ (Figure $1 \mathrm{~A})$. To determine whether increased $C X C L 10$ expression was associated with a type 1 pathway, we examined the correlation between IFNG and CXCL10 mRNA levels in asthma patients and found a moderate, statistically significant correlation $(\mathrm{r}=0.6115, P<0.0001)$ (Figure 1B). Using the healthy control mean and SD of 14.98, we established a cut-off of 60.46 ( 3 standard deviations above the 
A

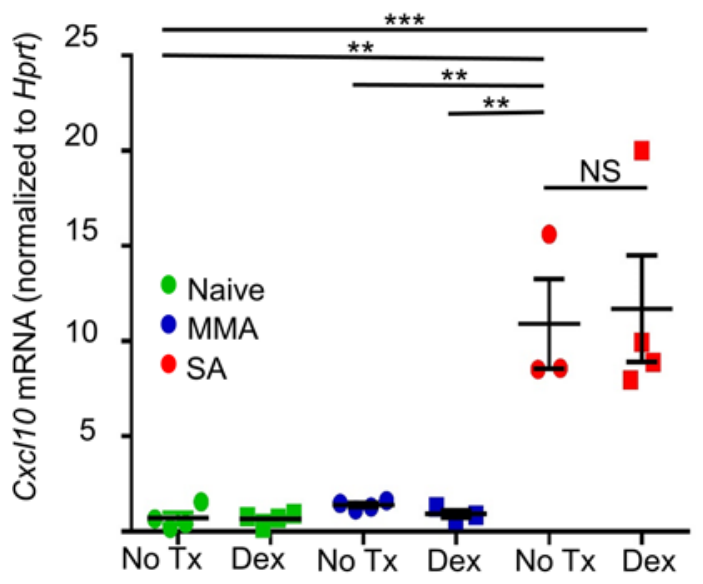

B

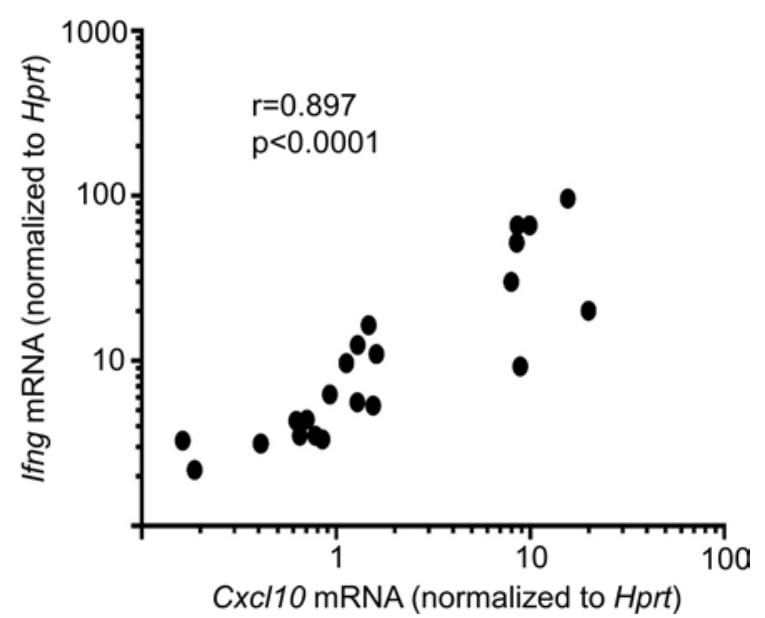

Figure 2. Cxc/10 expression is elevated in a mouse model of severe asthma and correlates with Ifng expression. (A) Cxc/10 mRNA was obtained from whole lung homogenates of BALB/c mice sensitized and challenged with house dust mite (HDM) only (MMA) ( $n=4$ placebo [No Tx], $n=3$ Dexamethasone [Dex]), or with HDM + cyclic-di-GMP (SA) ( $n=3$ No Tx, $n=4$ Dex) or were untreated mice (naive, $n=4$ both groups); all groups were treated with Dex or No Tx. (B) Cxc/10 mRNA and Ifng mRNA levels in whole lung homogenates were correlated across all mouse models $(n=22)$. Error bars represent \pm SEM, NS $P>0.05$, ${ }^{* *} P<0.01,{ }^{* *} P<0.001$ for Tukey's posthoc test for multiple comparisons (A). Spearman's nonparametric correlation was used in B.

healthy control mean) to divide asthmatic patients into 2 groups: CXCL10 Low ( $n=27,77 \%$ of population) and CXCL10 High ( $n=8,23 \%$ of population). When we assessed the relationship between CXCL10 and IFNG levels in these subgroups, we identified a strong correlation in the CXCL10 High group $(\mathrm{r}=0.9048, P$ $=0.005)$, while correlation in the CXCL10 Low group fell to a lower but still significant value $(\mathrm{r}=0.4695, P$ $=0.01$ ) (Figure 1C). After dividing patients into these groups, we then completed a post-hoc analysis using existing clinical data for the SARP I/II cohorts. A comparison of cell numbers and cell types in BAL revealed no significant differences, arguing that differences in CXCL10 expression were not related to variations in BAL cellular makeup or recovery (Supplemental Table 1; supplemental material available online with this article; https://doi.org/10.1172/jci.insight.94580DS1). We then evaluated baseline patient data and found no significant difference in age, race, age of onset, BMI, asthma quality of life questionnaires (AQLQ), IgE, fraction of exhaled NO (FeNO), or baseline spirometry (Table 2). We did observe a trend toward a lower percent increase in $\mathrm{FEV}_{1}$ in response to albuterol in the CXCL10 High group (percent increase of $8.28 \%[ \pm 2.95 \%]$ vs. $19.27 \%$ [ $\pm 3.15 \%$ ] for CXCL10 Low, $P=0.06$ ) (Figure 1D). As disease control is one of the key markers for SA, we next examined if there were markers of poor disease control associated with elevated CXCL10 expression levels. We found that the CXCL10 High group had a statistically significant increase in the proportion of patients who had experienced either an oral CS burst (OCS burst) $(85.7 \%$ vs. $26.2 \%$ for CXCL10 Low, $P<0.01)$ or emergency department (ED) visit for breathing $(83.3 \%$ vs. $21.7 \%$ for CXCL10 Low, $P=0.01)$ in the prior 12 months, both markers for asthma exacerbations (Figure 1E).

Cxcl10 mRNA levels are elevated in the lungs of mice exposed to a SA model and correlate with Ifng levels. Given the association of elevated CXCL10 mRNA levels with SA and poor disease control in the SARP I/II population, we hypothesized that increased expression of $C X C L 10$ may be a marker for poor CS response, and we sought to understand the effect of CS on CXCL10 expression. To accomplish this, we used our previously published mouse model of SA, which combined house dust mite (HDM) antigen with cyclic-di-GMP generating a high type 1 signature with increased airway resistance and poor response to the CS dexamethasone (Dex) (5). We found that mice exposed to this severe model expressed a higher level of Cxcl10 mRNA at the whole-lung level compared with mice subjected to a mild to moderate asthma model (MMA) involving sensitization and challenge with HDM only (mean Cxcl10 expression of 10.9 [ \pm 2.36$]$ for SA vs. $1.4[ \pm 0.10]$ for MMA, $P$ $<0.01$ ) (Figure 2A). We observed no significant difference in Cxcl10 expression after Dex treatment (mean Cxcl10 expression of $11.71[ \pm 2.80]$ for SA + Dex, $P>0.99$ ). Previously published work has shown that type 1 inflammation is critical to the asthma phenotype in this model, with absence of AHR in IFN- $\gamma^{1-}$ mice. Given the known importance of CXCR3 ligands, principally CXCL10, in establishing a type 1 signature in a tissue, blockade of the CXCR3 pathway is expected to generate similar data due to the loss of recruitment of 
Table 2. Patient characteristics for CXCL10 Low versus CXCL10 High groups

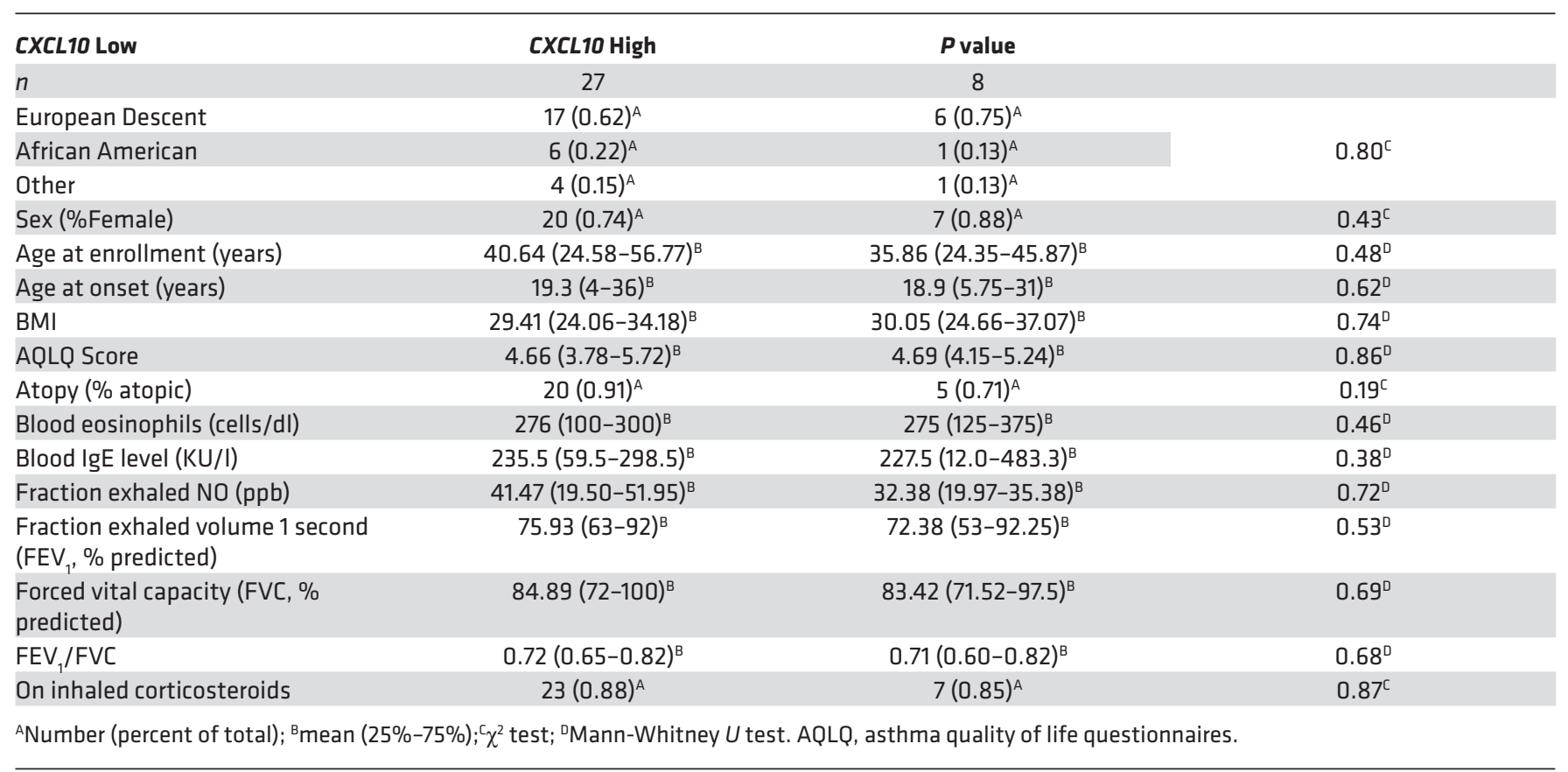

Th1 cells resulting in loss of AHR, as observed in the IFN- $\gamma^{-1-}$ mice. Indeed, levels of Ifng mRNA correlated positively with Cxcl10 mRNA levels in all mice ( $\mathrm{r}=0.897, P<0.0001)$ (Figure $2 \mathrm{~B}$ ). The combined human and mouse data allowed us to ask the central question in this study, which was whether high IFN- $\gamma$-driven $C x c l 10$ gene expression is refractory to CS and underlies poor disease management in a subset of severe asthmatics.

The low Cxcl10 expression in our MMA model appears to resemble the MMA asthma patients who express relatively lower levels of this chemokine. Prior work in other mouse models has also shown a role for CXCL10 in the context of type 2 inflammation in mice sensitized and challenged with OVA (26), with blockade of CXCR3 significantly improving AHR (27). This is consistent with additional data in humans showing that multiple stimuli can enhance Cxcl10 expression (11) and that increased levels of CXCL10 protein were detected in humans subjected to segmental allergen challenge (20). Notably, both of these type 2-associated CXCL10 elevations were in the absence of a prominent type 1 signature. It has been suggested that CXCL10 may also promote the recruitment of type 2 lymphocytes to the airways, even though type 2 cells express low levels of CXCL10 $(26,27)$

IFN-y-but not LPS-mediated induction of CXCL10 mRNA and protein is resistant to suppression by CS. To better understand the etiology of insensitivity of the IFN- $\gamma / C X C L 10$ pathway to CS, we adopted an in vitro system. THP-1 cells were treated with Dex overnight, and the cells were then exposed to 2 known inducers of CXCL10: IFN- $\gamma$ - which activates STAT1, resulting in binding to the IFN-stimulated response element (ISRE) and the IFN- $\gamma$ activated sequences (GAS) in the $C X C L 10$ promoter - and LPS acting via NF- $\kappa$ B can bind to $\kappa B 1 / \kappa B 2$ promoter sites, which are highly conserved in the murine and human promoters (14-16). The ISRE element is central to $C X C L 10$ promoter activation by IFN- $\gamma(14)$ and can be also utilized by LPS (14). However, the $\kappa B$ sites alone can confer LPS-mediated promoter activation (14). CXCL10 mRNA levels were markedly increased by both LPS and IFN- $\gamma$ treatments (mean CXCL10 fold change of 382.9 [ \pm 50.43$]$ for LPS and 470.8 [ \pm 128.5$]$ for IFN- $\gamma, P=0.92$ for comparison between IFN- $\gamma$ and LPS induction). There was no significant change in IFN- $\gamma$-mediated induction of CXCL10 mRNA levels with Dex pretreatment (mean CXCL10 fold change 645.6 [ \pm 91.46$]$ for combined exposure to IFN- $\gamma$ and Dex, $P=0.49$ ). However, LPS-mediated induction of $C X C L 10$ was markedly lowered by Dex pretreatment compared with IFN- $\gamma+$ Dex treatment (CXCL10 fold change of 173.1 [ \pm 20.77$]$ for combined treatment with LPS and Dex, $P<0.01$ ) (Figure 3A). To determine whether the failure to suppress IFN- $\gamma$-mediated induction of $C X C L 10$ was specific to Dex, we also pretreated cells with fluticasone propionate (FP) and did not observe a significant effect on IFN- $\gamma$-mediated CXCL10 induction (mean $C X C L 10$ fold change of 451.8 [ \pm 84.3 ] for IFN- $\gamma$ vs. 286.3 [ \pm 27.32$]$ for IFN- $\gamma+$ FP, $P=0.11$ ) (Figure 3B). 


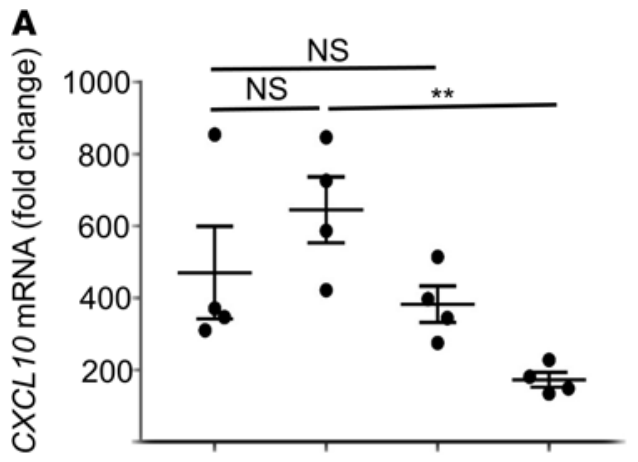

IFN-y IFN- -+ Dex LPS LPS+Dex

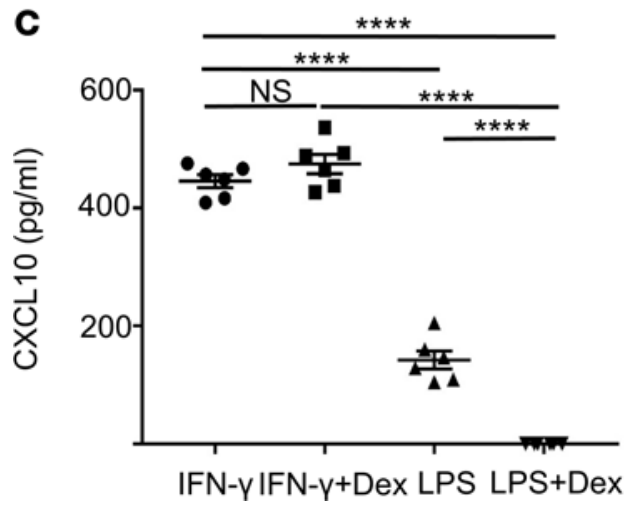

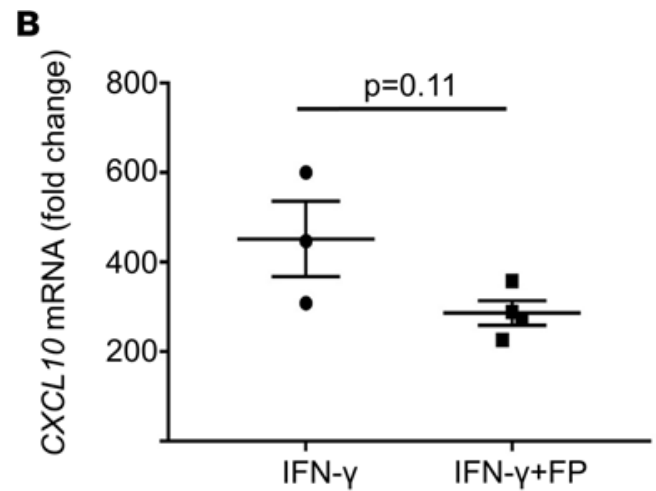

D

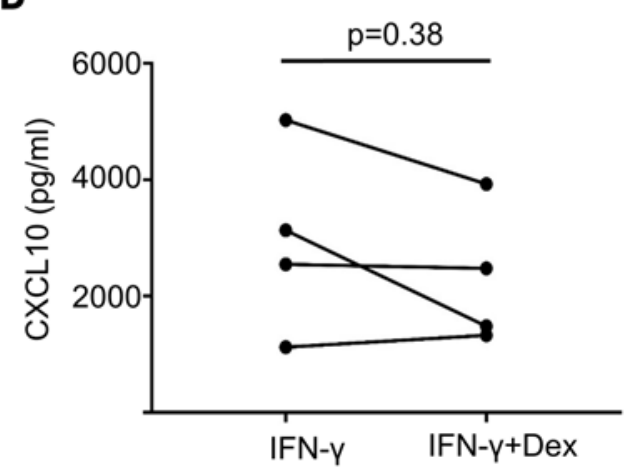

Figure 3. CXCL10 induction by IFN- $\gamma$ is not inhibited by CS (Dex) treatment. (A) THP-1 cells were cultured overnight with dexamethasone (Dex) or without and then exposed to LPS or IFN- $\gamma$. The cells were harvested after 3 hours and CXCL10 mRNA levels determined (data shown pooled from 3 separate experiments). (B) Cells were treated as in A, but fluticasone propionate (FP) was used in place of Dex, and CXCL10 mRNA levels were assessed (data representative of 2 separate experiments). (C) Cells were treated as in $\mathbf{A}$, with supernatant collected 24 hours after stimulation and CXCL10 protein levels determined by ELISA (data representative of 4 separate experiments). (D) Human monocytes were isolated from leukopaks $(n=4)$ using CD14 magnetic bead isolation and treated as in C, with CXCL10 levels determined by ELISA $(n=4)$. Error bars show \pm SEM, ${ }^{* *} P<0.01,{ }^{* * *} P<0.0001$ by Tukey's posthoc test $(\mathbf{A}$ and $\mathbf{C})$, Mann-Whitney $U$ test (B), and Wilcoxon matched pairs (D).

We next examined whether our findings at the transcript level were also true at the protein level. Cells were treated as above, and culture supernatants were analyzed for CXCL10 protein levels. Despite similar CXCL10 mRNA induction by IFN- $\gamma$ and LPS, IFN- $\gamma$ induced much higher levels of CXCL10 protein compared with LPS (mean CXCL10 445.7 [ \pm 11.02$] \mathrm{pg} / \mathrm{ml}$ for IFN $-\gamma$ compared with $142.4[ \pm 15.25] \mathrm{pg} / \mathrm{ml}$ for LPS, $P<0.0001)$. As discussed below, prior studies have shown remarkable stabilization of IFN- $\gamma$-induced but not LPS-induced $C X C L 10$ mRNA $(28,29)$. There was no significant effect on IFN- $\gamma$-induced CXCL10 protein levels by Dex (mean CXCL10 $474.6[ \pm 16.44] \mathrm{pg} / \mathrm{ml}$ for IFN- $\gamma+$ Dex, $P=0.38)$. In the presence of Dex, LPS-induced CXCL10 protein levels were suppressed below the lower limit of detection $(P<0.0001)$ (Figure $3 \mathrm{C})$. To confirm our findings in primary cells, we examined the effect of Dex on IFN- $\gamma$-induced CXCL10 protein levels in primary human monocytes isolated from leukopaks $(n=4)$. Despite prior exposure to Dex, stimulation with IFN- $\gamma$ led to CXCL10 protein levels of $>1 \mathrm{ng} / \mathrm{ml}$ in all culture supernatants, with no significant difference between IFN- $\gamma$ and IFN $-\gamma+$ Dex treatments $(P=0.38$ for matched pairs) (Figure 3D).

$I F N-\gamma$ does not impair cellular response to Dex in THP-1 cells or in primary human monocytes. We next sought to answer the question of whether the failure of Dex to inhibit CXCL10 gene expression was due to impairment of the cellular response to CS caused by IFN- $\gamma$. Primary human monocytes were treated with IFN- $\gamma$ in the presence or absence of Dex. Cells were then fixed and processed for imaging cytometry to identify the GR in the nuclear and cytoplasmic compartments. There was a shift in the similarity score upon Dex treatment, indicating GR nuclear translocation, which was also evident in the image (Figure 4A and Supplemental Figure 1). Importantly, this shift was not impaired by the presence of IFN- $\gamma$ (Figure 4A). Interestingly, treatment with IFN- $\gamma$ alone also induced an increase in similarity score, implying the ability of IFN- $\gamma$ alone to promote nuclear localization of GR (Fig- 
A

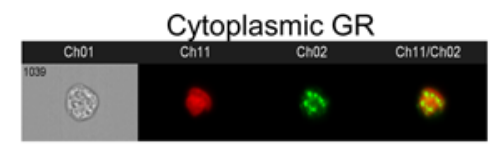

Nuclear GR

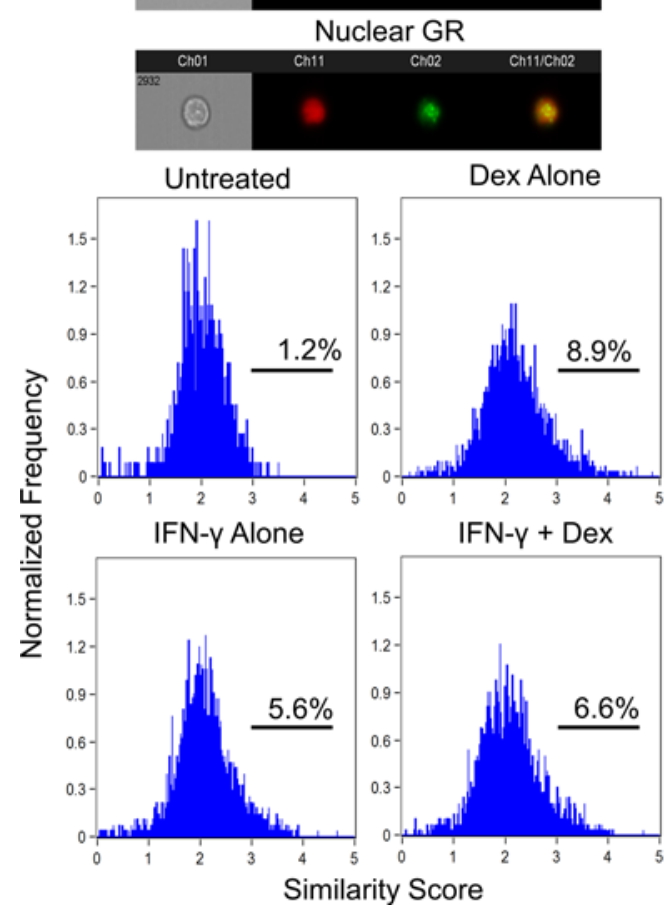

B

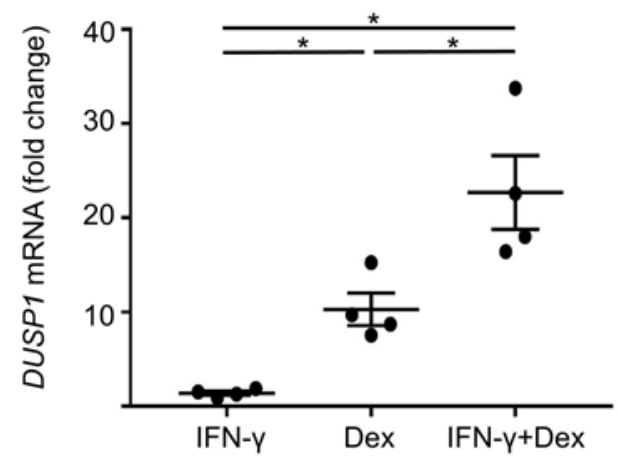

C

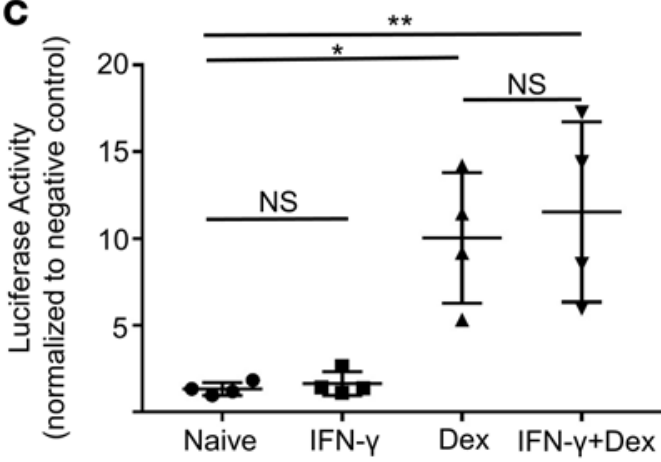

Figure 4. IFN- $\gamma$ does not suppress the cellular response to CS. (A) Human PBMCs were treated with IFN- $\gamma$, Dexamethasone (Dex), or both for 1 hour and were analyzed by imaging cytometry using an ImageStreamX Mark II instrument. Gating on monocytes was accomplished using cell area versus aspect ratio, with verification by CD14 staining. Similarity scores (the degree of glucocorticoid receptor [GR] and nuclear counterstain, DRAQ5 colocalization) were calculated for each condition using the Amnis IDEAS software nuclear translocation wizard. Using visual assessment of cytoplasmic versus nuclear GR (representative examples shown) and comparing untreated with treated samples, final gating was set on the similarity score versus normalized frequency plots. Shown are images of brightfield (channel 1), nuclear (DRAQ5) staining (channel 11), GR staining (channel 2), and merged images of nuclear and GR staining (channels 11 and 2). The values shown are the percentage of the monocyte population in which GR has been translocated to the nucleus. (B) Human monocytes were treated as in A and harvested after 3 hours for analysis of DUSP1 mRNA levels (data pooled from 2 separate experiments). (C) THP-1 cells underwent nucleofection with a plasmid containing a glucocorticoid response element (CRE) linked to the luciferase gene. Luciferase activity detected by a luminometer was normalized to Renilla luciferase activity used as a transfection control (data shown representative of 2 separate experiments). Error bars show $\pm \mathrm{SEM},{ }^{*} P<0.05,{ }^{* *} P<0.01$ Tukey's posthoc test (B-D).

ure 4A). To exclude nuclear sequestration of unliganded GR as a mechanism for steroid insensitivity, we next examined expression of a downstream target of GR, dual-specificity phosphatase 1 gene (DUSP1), which is a well-recognized driver of the antiinflammatory effects of CS (30). We observed a significant induction of DUSP1 with Dex compared with that induced with IFN- $\gamma$ alone (DUSP1 fold change of $1.40[ \pm 0.21]$ for IFN- $\gamma$ vs. $10.29[ \pm 1.70]$ for Dex, $P=0.02)$. The addition of IFN- $\gamma$ to Dex caused a further increase in DUSP1 expression (DUSP1 fold change of 22.68 [ \pm 3.92$], P=0.02$ compared with IFN- $\gamma$ and $P=0.04$ compared with Dex) (Figure 4B). Finally, to assess the impact of IFN- $\gamma$ on transactivational functions of GR, we utilized a reporter assay using a plasmid construct containing the glucocorticoid response element (GRE) promoter linked to the reporter gene luciferase. After nucleofection, THP-1 cells were exposed to IFN- $\gamma$ alone, Dex alone, or the combination, and luciferase activity was measured. No significant induction of luciferase activity was observed with IFN- $\gamma$ alone, but, as expected, Dex induced a marked increase in activity, which was unchanged in the presence of IFN- $\gamma$ (Figure 4C). Taken together, these results showed an intact cellular response to CS with appropriate GR translocation, transcription of a GR-target gene, and transactivational function of GR in the presence of IFN- $\gamma$.

No change in STAT1 phosphorylation in the presence of Dex, but corecruitment of STAT1 and GR to ISRE and GAS elements in the CXCL10 promoter. THP-1 cells were either pretreated with Dex as before, simultaneously treated with Dex in the presence or absence of IFN- $\gamma$, or left untreated (last lane). Levels of STAT1 phosphorylation at 2 sites, tyrosine 701 (Tyr701) and serine 727 (Ser727), were examined 
A

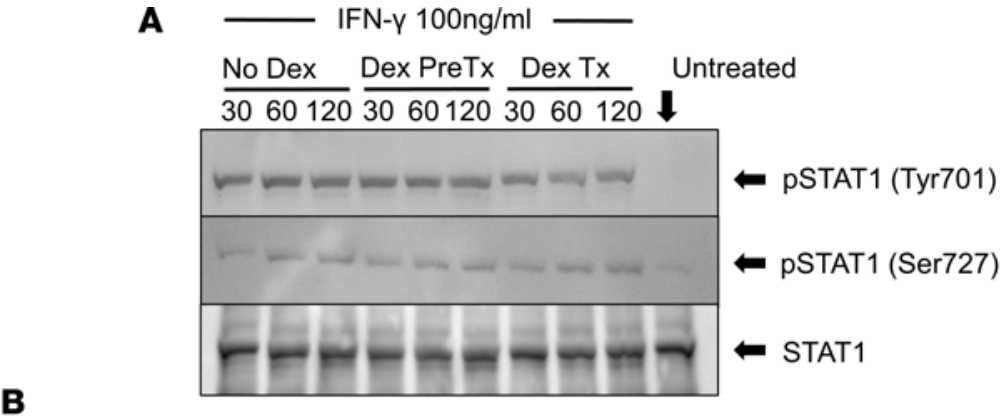

B
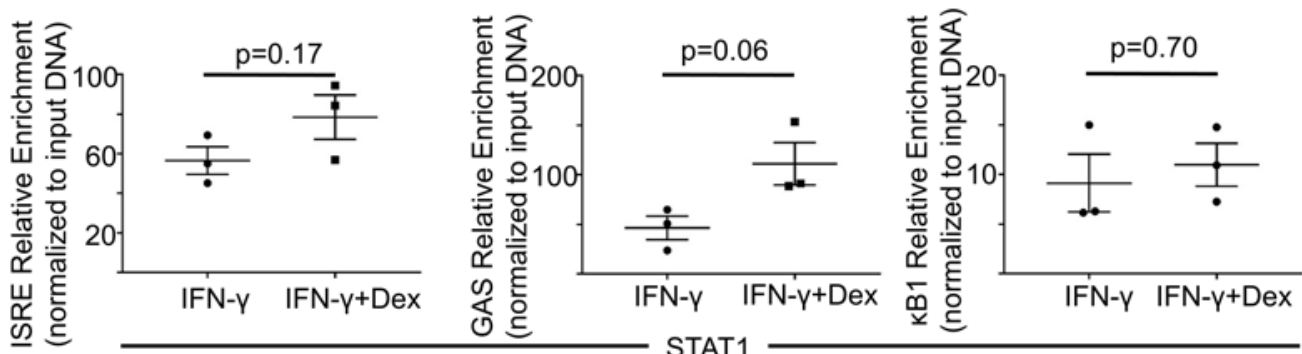

C

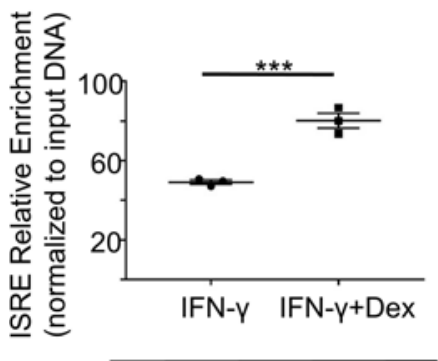

STAT1

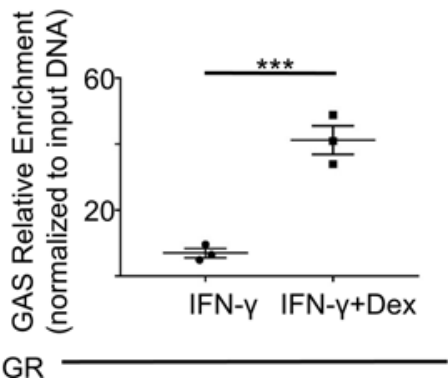

D

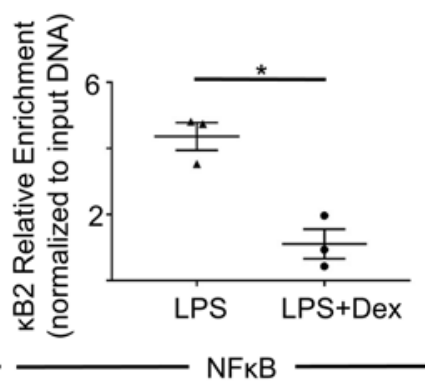

Figure 5. Similar STAT1 phosphorylation induced by IFN- $\gamma$ and Dexamethasone (Dex) and corecruitment of STAT1 and the glucocorticoid receptor (GR) to the CXCL10 promoter. (A) THP-1 cells were left untreated (No Dex) or treated with Dex overnight (Dex PreTx) or together with IFN- $\gamma$ (Dex Tx) and then exposed to IFN- $\gamma$ for 30, 60, or 120 min. The cells were then lysed and STAT1 total protein, and phosphorylation at Tyrosine 701 (Tyr701) and Serine 727 (Ser727) were detected by Western blotting and compared with total STAT1 levels (blot representative of 2 separate experiments). (B) THP-1 cells were treated with IFN- $\gamma$ alone, or IFN- $\gamma$ was added after pretreatment with Dex. Cells were lysed after 3 hours. ChIP was performed using anti-STAT1 Ab followed by PCR across the IFN sensitive response element (ISRE), IFN- $\gamma$ activated sequence (CAS), and NF- $\kappa B 1$ sites in the CXCL10 promoter (data shown representative of 3 separate experiments). (C) ChIP was performed using anti-GR Ab followed by PCR across the ISRE and GAS promoter sites (data shown representative of 2 separate experiments). (D) THP-1 cells were treated with LPS alone or with LPS after pretreatment with Dex. Cells were lysed after 3 hours, and ChIP was performed using anti-RelA Ab followed by PCR across the NF- $\kappa$ B2 site in the promoter (data represents 3 separate experiments). Error bars show mean and \pm SEM, Mann-Whitney $U$ test (B-E).

by Western blot analysis methods. IFN- $\gamma$ induced STAT1 phosphorylation at both sites, with Tyr701 phosphorylation slightly decreasing after 30 minutes of IFN- $\gamma$ treatment and the Ser727 phosphorylation increasing with exposure time; however, no difference between any treatments was noted (Figure 5A and Supplemental Figure 2; see complete unedited blots in the supplemental material).

We next examined the status of STAT1 and GR binding to the ISRE and GAS elements in the CXCL10 promoter in the presence of IFN- $\gamma$ alone or IFN- $\gamma$ + Dex. THP-1 cells were treated as before and were then lysed for use in ChIP assay using anti-STAT1 antibody (Ab). qPCR across both the ISRE and GAS promoter sites revealed increased enrichment at both sites, and it was similar in the presence or absence of Dex (Figure 5B). As a negative control, we also performed qPCR across the $\kappa B 1$ site after ChIP with anti-STAT1 Ab, showing minimal recruitment to this site (Figure 5B). We then repeated this experiment using anti-GR $\mathrm{Ab}$ and performed qPCR across the same sites. Here, we observed significant recruitment of GR to the ISRE site with IFN- $\gamma$ alone (Figure 5C). This matched our image cytometry data where we had noticed an increase in similarity score with IFN- $\gamma$ treatment alone, indicating an increase in GR nuclear translocation in the presence of IFN- $\gamma$ (Figure 4A). The recruitment of GR to the GAS site was less evident 
with IFN- $\gamma$ treatment alone (Figure 5C). However, as expected, Dex treatment caused a significant increase in relative enrichment of GR at both the ISRE and GAS sites, and the difference between treatment with IFN- $\gamma$ alone and IFN- $\gamma+$ Dex reached statistical significance $(P=0.001)$ (Figure $5 C)$. Given the ability of Dex to suppress LPS-induced cxc110 mRNA expression, we also performed ChIP using an Ab against RelA (the p65 subunit of NF- $\mathrm{kB}$ ), and qPCR across the $\kappa \mathrm{B} 2$ site showed a significant decrease in relative enrichment when cells were treated with Dex before LPS stimulation $(P=0.03)$ (Figure 5D).

CXCL10 and type 1 signatures correlate with mast cell presence in asthma. As CXCL10 is known to signal through the cognate receptor CXCR3 (11), we investigated correlation between $C X C L 10$ and $C X C R 3$ in microarray data previously obtained from patients in the SARPI/II cohorts. Indeed, we detected a moderate and significant positive correlation between the $2(\mathrm{r}=0.596, P<0.0001)$ (Figure 6A). Given evidence of mast cell accumulation in the airway submucosa of SA patients (31), in airway smooth muscle cells (ASMs) of some asthmatics (17), and also in the sputum of patients with persistent asthma (32), we examined if there was any correlation between CXCL10 mRNA levels and mast cell markers. Using BAL cell-derived mRNA, we assessed levels of the gene carboxypeptidase A3 (CPA3) and tryptase, 2 genes associated with mast cells, and observed a significant positive correlation for both genes ( $\mathrm{r}=0.446, P=0.008$ for $C P A 3$, and $\mathrm{r}=0.467, P=0.005$ for tryptase), suggesting an influence of CXCL10 on mast cell presence in the airways (Figure 6B). Based on these findings, we then examined mast cell presence in the airways of mice subjected to the SA or milder asthma (MMA) models. Staining with toluidine blue showed no significant mast cell presence in the airways of untreated mice, but it readily revealed mast cells in the airways of mice subjected to both mild moderate and SA models. The mast cell numbers were not affected by Dex in either model (Figure 6C, representative images, $n=4$ per group).

\section{Discussion}

Although CS are the mainstay of asthma treatment and the defining therapy for SA (33), it was previously shown that percent of forced expiratory volume in 1 second $\left(\% \mathrm{FEV}_{1}\right)$ in patients with $\mathrm{Th} 2^{\text {low }}$ signature, unlike those with $\mathrm{Th} 2^{\text {high }}$ profile, does not respond to CS therapy (4). This observation was insightful, drawing attention to both Th2 and non-Th2/non-type 2 mechanisms affecting lung function, which did not respond to standard inhaled CS therapy. While disease severity in these CS-unresponsive subjects was not discussed in the study, it is well known that $5 \%-10 \%$ of asthma patients suffering from SA do not respond adequately to CS therapy (2, 8). These subjects experience frequent exacerbations and poor disease control, and they typically bear $50 \%$ of healthcare burden for all asthma (8, 34-36). It is now generally accepted that asthma is not a single entity, but a collection of multiple phenotypes, each with its own natural history, pathobiology, and unique response to therapy $(2,8)$. Our understanding of the mechanisms underlying poor response to steroid-refractory disease is limited, and it was investigated in the present study. We recently reported an elevated type 1 signature marked by IFN- $\gamma$ in approximately $50 \%$ of patients with SA (5). Furthermore, using our mouse model of SA, we showed that elevated IFN- $\gamma$ leads to high airway resistance, increased inflammatory infiltrates in the lung, and poor response to CS treatment (5). We have also recently documented a higher level of expression of the transcription factor IRF5 in the lungs of severe asthmatics compared with that in their milder counterparts and demonstrated a critical role for this molecule, expressed by lung DCs and macrophages, in regulation of the Th1/IFN- $\gamma$ response in our SA model (37). In the present study, we asked whether IFN- $\gamma$ contributes to CS unresponsiveness by focusing on its downstream target CXCL10, the best described of several CXCR3 ligands that serve to recruit Th1 cells as well as mast cells and eosinophils to the site of inflammation (11). Given the role of CXCL10 in Th1 cell recruitment, and thus promoting a feed-forward loop to promote type 1 inflammation, we hypothesized that the inability of CS to inhibit CXCL10 induction would limit their usefulness in breaking this persistent type 1 inflammatory pattern, thereby rendering CS a poor treatment choice for the control of immune dysfunction and its sequelae in severe Th $1^{\text {high }}$ disease. Here, we show relatively high levels of CXCL10 mRNA in the BAL cells of $50 \%$ of SA subjects and also in the airways of mice subjected to a SA model in the context of high dose CS treatment. CS and IFN- $\gamma$ together elicited recruitment of both the GR and STAT1 to the CXCL10 promoter in monocytes, and this was associated with unabated CXCL10 expression at both mRNA or protein levels in the cells. High CXCL10 expression was also associated with a mast cell signature in both humans and mice.

The expression of CXCL10 mRNA has been previously documented in asthma in BAL cells (21) and in infiltrated cells in the submucosa in the airways (22). Most prior studies of CXCL10 in asthma have focused on atopic and mild disease $(17,21,23)$. Interestingly, CXCL10 protein level was found to increase significantly upon segmental allergen challenge in humans (20). Studies in mouse models with type 2-dominated inflammation have also documented an increase in CXCL10 levels in the airways regulating AHR and 


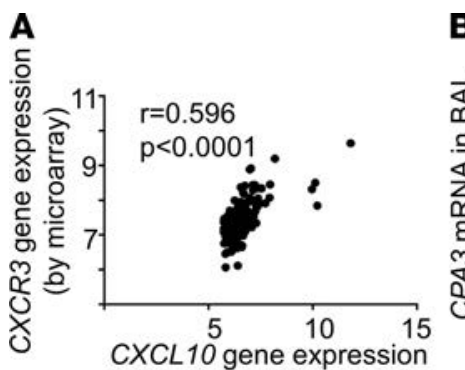

(by microarray)
B

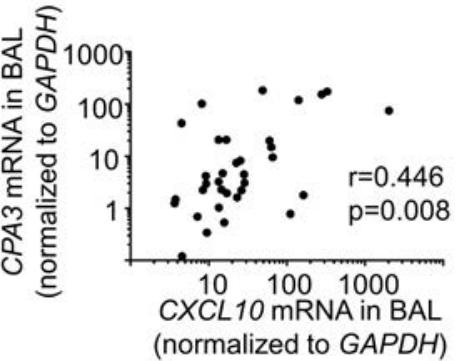

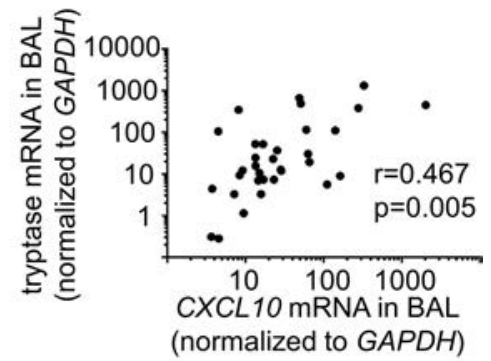

C

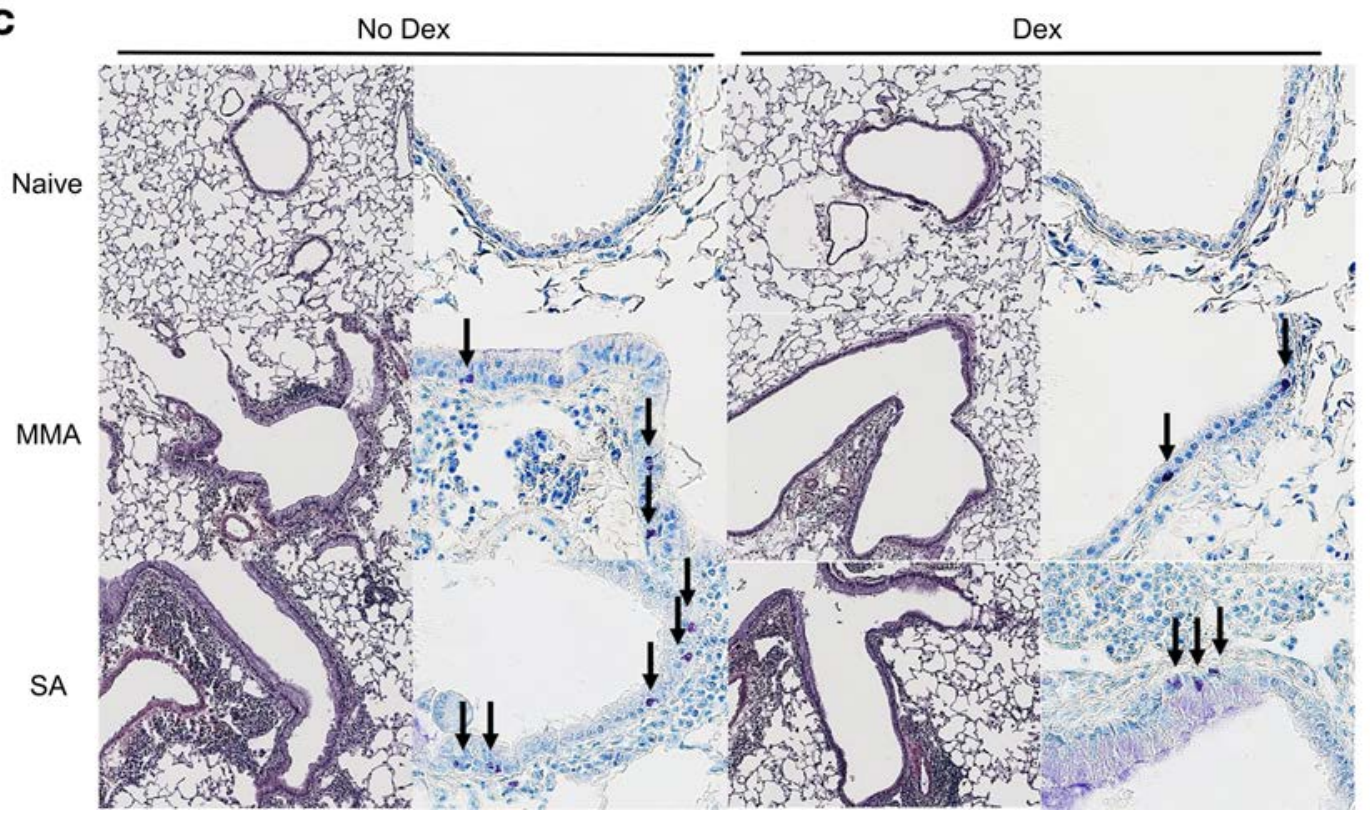

Figure 6. CXCL10 expression associates with expression of mast cell proteases and mast cell presence in Th1-driven asthma. (A) Bronchial epithelial cell brushings were subjected to microarray analysis, and correlation between expression of CXCR3 and CXCL10 is shown ( $n=155)$. (B) Carboxypeptidase A3 (CPA3) $(n=34)$ and tryptase $(n=35)$ mRNA levels in BAL cells from patients with asthma compared with CXCL10 expression. (C) Mice were treated as shown in Figure 2, and lungs sectioned for histological assessment ( $n=4$ per group). Lung sections were stained with H\&E (left panel, 10× ) or toluidine blue (right panel, 20x). Arrows show mast cells stained violet (representative sample shown). Spearman's correlation (A and B).

presumably recruitment of $\mathrm{T}$ cells to the airways $(26,27)$. Taken together, these findings show the ability of multiple stimuli to induce CXCL10, although the levels may be higher in the setting of type 1 inflammation, as our data show by using both human samples and mouse models. However, it will be important to determine the expression characteristics of all of the family members, as well as those of CXCR3 in the infiltrated T cells, in additional studies in both human disease and mouse models. A previous study showed an association between elevated CXCR $3^{+} \mathrm{CD} 4^{+} \mathrm{T}$ cells in the blood and asthma severity (38), but ours is the first to show a strong presence of CXCL10 in BAL cells of SA subjects and to also highlight a positive correlation between high CXCL10 levels and markers of poor disease control (OCS burst and ED use in the prior 12 months). Furthermore, we have found that the correlation between CXCL10 and IFNg is stronger within the CXCL10 High group, suggesting that other inducers may be involved in inducing a lower level of CXCL10 expression in mild to moderate asthma. It should be emphasized that disease control assessment is limited by post-hoc analysis in our study, and further prospective clinical outcomes data, as well as longitudinal assessment of the permanency of CXCL10 levels in human subjects, is needed. It is important to note that, while CXCR3 is a well-recognized receptor for CXCL9/10/11 and has been implicated both in Th1 priming (39) and recruitment of IFN- $\gamma$-secreting cells to the site of inflammation (40-42), there are other molecules like CCR5 that can also promote Th1 cell chemotaxis (43). Therefore, an additional complexity in regulation of inflammatory cell recruitment and potentially response to therapy lies in the sensitivity of chemokine receptor expression to CS, which would be interesting to investigate in future studies. 
In the context of human rhinovirus infection, which is frequently associated with asthma exacerbations, both epithelial cells and monocytes were found to be important sources of CXCL10; however, monocytes appear to be the dominant cell type (25). Additionally, studies comparing CXCL10 production by monocytes versus alveolar macrophages have documented similar chemokine production by the 2 cell types, and peripheral blood monocytes are considered appropriate cells to study CXCL10 gene expression (24, 44). Having observed increased expression of $C X C L 10$ in the BAL cells of a subset of severe asthmatics correlating strongly with IFNG expression, and given the utility of monocytes to study mechanisms of CXCL10 gene expression, we used the human monocytic cell line THP-1 as well as peripheral blood monocytes to assess CXCL10 mRNA and protein expression in the context of IFN- $\gamma$ and CS. Given elevations of CXCL10 levels in type 2 -associated inflammatory states in mouse models of asthma $(26,27)$, as well as in mild-moderate atopic asthma as described above $(17,21,23)$, we hypothesized that IFN- $\gamma$-mediated induction of CXCL10 might be uniquely insensitive to CS compared with when it is induced by other stimuli. LPS is also a well-studied inducer of CXCL10, but there are differences in CXCL10 promoter utilization between IFN- $\gamma$ and LPS (14). Both STAT1- and NF-kB-binding sequences are present in the CXCL10 promoter, which mediates gene transcription in response to IFN- $\gamma$ and LPS. Most interestingly, previous studies have shown that IFN- $\gamma$, but not LPS, stabilizes CXCL10 mRNA via MyD88, although LPS utilizes MyD88 for signaling downstream of TLR4 $(28,29)$. In line with these previous reports, our ongoing studies show that, while both IFN- $\gamma$ and LPS can induce CXCL10 gene transcription, IFN- $\gamma$ stimulation causes $>3$ - to 4-fold more CXCL10 protein production from THP-1 cells compared with that induced by LPS. At both mRNA and protein levels, expression induced by IFN- $\gamma$ is largely insensitive to CS treatment, while that induced by LPS can be suppressed. This is important, as it emphasizes that as a rule, the expression of CXCL10 and likely of other CXCR3 ligands is not CS sensitive or CS insensitive, but rather the mediator involved in their induction is critical in determining response to this treatment modality. Given the continuum of CXCL10 levels seen in our cohort, we suspect that there is overlap between type 1 and non-type 1 induction of CXCL10 at the margins, and this may explain the different clinical response of mild-moderate asthma patients with slightly elevated levels compared with SA patients with markedly elevated levels of the chemokine.

Our ChIP data provide a mechanistic basis for the insensitivity of IFN- $\gamma$ - but not LPS-induced CXCL10 transcription to CS exposure and, as such, helps to address this question of CXCL10 association with both mild-moderate asthma and SA, as these are likely mediated through different inducers. It has been previously shown that the ISRE in the proximal promoter in the murine $C x c 110$ gene is critical for IFN- $\gamma$-induced promoter activation (14). In addition, any of the $2 \mathrm{NF}-\mathrm{kB}$-binding sites in the proximal promoter can function in conjunction with the ISRE site to induce promoter activation in the presence of IFN- $\gamma$ (14). These ISRE and NF- $\mathrm{KB}$ sites are conserved in the human $C X C L 10$ promoter and have been also implicated in transcriptional activation by rhinovirus, IFN- $\gamma$, and LPS $(15,16)$. Since IFN- $\gamma$-induced STAT1 homodimer can also bind to GAS, we also examined recruitment of STAT1 to one of the GAS elements in the CXCL10 promoter. IFN- $\gamma$-induced STAT1 binding to both ISRE and GAS sequences was not only unaffected in the presence of Dex, but it was actually slightly enhanced, and this pattern matched IFN- $\gamma$-induced CXCL10 mRNA and protein levels in the presence or absence of Dex. Using anti-GR Ab, we detected GR recruitment to both the ISRE and GAS elements in the presence of IFN- $\gamma$ and Dex. Surprisingly, GR enrichment on these sites was also observed with IFN- $\gamma$ alone, particularly on the ISRE element. While constitutive nucleocytoplasmic shuttling of the GR in the absence of CS has been documented (45), in THP-1 cells, no basal recruitment of GR to the GAS site was detectable (data not shown). However, the results of the ChIP and imaging cytometry experiments show that IFN- $\gamma$ alone in the absence of CS can promote nuclear translocation of GR with binding to the ISRE regulatory element in the CXCL10 promoter, which has not been reported previously. However, it is important to note that, without addition of CS (Dex), GR is not activated, as observed in our reporter assay. This raises the question of whether, without administration of exogenous CS, high levels of IFN- $\gamma$ in the airways may cause CXCL10 promoter activation via effects of endogenous CS such as cortisol. When LPS was used as a stimulus, we detected enrichment of the RelA (p65) subunit of NF- $\mathrm{\kappa B}$ on the $\kappa \mathrm{B} 2$ site of the CXCL10 promoter, but this was blunted to almost basal levels with Dex. We had previously shown that GR interacts with RelA and antagonizes its transactivation function, establishing GR-NF- $\mathrm{B}$ interaction as an important antiinflammatory effect of CS (46). Thus, in

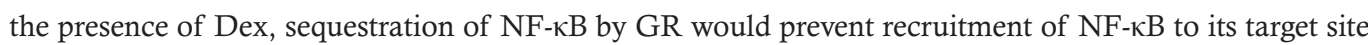
in the $C X C L 10$ promoter, as evident in the ChIP assay. Collectively, our data show that IFN- $\gamma$ alone causes both STAT1 and GR to bind to target sequences in the CXCL10 promoter, and exposure to CS allows this 
complex to maintain or even enhance IFN- $\gamma$-induced CXCL10 transcription. These data suggest that although different stimuli can enhance CXCL10 gene transcription, resulting in an increase in CXCL10 protein levels, as has been also documented in mild asthma (20-22) - without type 1 inflammation and STAT1 activation, the induced expression can most likely be inhibited by CS. Even if CXCL10 levels are not sufficiently inhibited by CS in milder disease, impact on disease may not be as dire without Th1 ${ }^{\text {high }}$ immune status. Cooperation between STAT1 and GR was also shown to mediate transcriptional activation of the Fc $\gamma \mathrm{RI}$ gene in monocytes (47). Engagement of Fc $\gamma \mathrm{RI}$ on monocytes promotes expression of the immunosuppressive gene IL-10 (48), and thus in this situation, STAT1-GR cooperation may actually support the antiinflammatory function of CS. Since an increase in Th1 response is desirable during pathogen invasion, and SA has been associated with both bacterial and viral infections (8), this attempt to fortify a Th1 response via increased production of CXCL10 unfortunately causes collateral damage in asthma.

A previous study implicated competition for the coactivator GRIP1 between the GR and IRF proteins in CS insensitivity in ASMs (49). GRIP1 is an important coactivator for transactivational functions of GR (50), and this coactivator is also utilized by multiple IRF family proteins to induce gene transcription (51). It was shown that, in ASMs, sequestration of GRIP1 by IRF-1 induced in response to a mixture of IFN- $\gamma$ and TNF- $\alpha$ results in reduced expression of DUSP1 (also known as MKP1), which is also associated with antiinflammatory functions of CS $(30,49)$. However, in our experiments, we did not observe any decrease in Dex-induced DUSP1 expression in the THP-1 cells in the presence of IFN- $\gamma$. If GR function were inhibited, we would have also observed inhibition of reporter gene activity, which was not the case. It is also possible that GRIP1 is not used by GR as a coactivator in monocytes, which will be interesting to determine in the future. By the same token, given that we did not observe any decrease in reporter gene activity in the presence of IFN- $\gamma$, it is unlikely that the non-hormone-binding splice isoform of GR - GR $\beta$, which has dominant-negative effects over GR $\alpha$ (52) - is involved in CS unresponsiveness in the presence of IFN- $\gamma$. It is also important to note that DUSP1 dephosphorylates MAPK and should not impact pSTAT1, which is dephosphorylated by SHP2 (53). Thus, even though this antiinflammatory arm is strengthened in the presence of IFN- $\gamma$ and CS, CXCL10 gene expression remains uncontrolled by CS.

We also investigated whether IFN- $\gamma$-induced CXCL10 is associated with mast cell infiltration of the airways of severe asthmatics. Our previous studies showed accumulation of mast cells in the airways of severe asthmatics (31), and more recently, they were detected in the sputum of subjects with persistent asthma (32). Their recruitment to ASMs in the airways of asthmatics was shown to require CXCR3 expressed by the cells (17). Despite a modest type 2 signature in our SA mouse model (previously published data; ref. 5), in the present study, we observed significant mast cell infiltration of the airways that was unaffected by Dex. In a study just published, treatment of severe asthmatics with imatinib - which targets KIT, a molecule expressed by mast cells - reduced AHR, even though mast cells numbers were reduced (but not eliminated) only after prolonged treatment (54). Mast cells are known to be long-lived, and given that they can contribute to AHR, the potential for type 1 inflammation to promote their accumulation in the airways highlights another detrimental effect of IFN- $\gamma$ in establishment of SA and the poor response of this disease state to CS. As expected, we also detected mast cells in the airways of mice subjected to the mild asthma model, and the degree of accumulation was comparable between the 2 models. At this time, the mouse data represent a qualitative evaluation, and a more careful quantitative estimate will be undertaken in future studies. Given that a moderate correlation between the expression of IFN- $\gamma$ and of the mast cell protease genes CPA3 and tryptase was noted in humans, it seems plausible that in mice, too, IFN- $\gamma$ is involved in their accumulation in the airways. While it is currently unknown whether murine mast cells also express CXCR3, a previous study of chronic asthma in mice documented IFN- $\gamma$-mediated mast cell recruitment to the airways mediated by IFN- $\gamma$ receptor 1 (IFN$\gamma \mathrm{RI}$ ) (55). Our previous study showed an inverse relationship between expression of IFN- $\gamma$ and the serine protease inhibitor, secretory protease leukocyte inhibitor (SLPI), in the airways of both humans and mice (5). SLPI is a potent inhibitor of tryptase and chymase. Tryptase can induce AHR by activating protease-activated receptor 2 (PAR-2), which is expressed on ASMs and has been implicated in bronchoconstriction through release of neurokinins from afferent neurons in the airways (56).

Taken together, our study demonstrates elevated CXCL10 signature in a subgroup of severe asthmatics that correlates with markers of poor disease control. When viewed in aggregate, the IRF5/IFN- $\gamma /$ CXCL10 pathway appears to be steroid refractory $(5,37)$. Based on ChIP data, once established, this pathway may actually be perpetuated by chronic steroid therapy through interactions between GR and STAT1 on target sites in the CXCL10 promoter. As observed in our study using monocytic cells, in a previous study of air- 
way epithelial cells derived from patients with chronic obstructive pulmonary disease, CS did not inhibit IFN- $\gamma$-induced STAT1 phosphorylation (57). This inability of CS to inhibit STAT1 phosphorylation can be expected to facilitate GR-STAT1 collaboration, as observed in our ChIP assay. Thus, our findings suggest that not only are CS ineffective in controlling the underlying inflammatory state in these patients, but they may actually make the disease worse over time by further stabilizing STAT1 promoter interactions, leading to increased CXCL10 production. Increased levels of CXCL10 would induce the recruitment of additional Th1 cells, leading to persistence of type 1 inflammation. Furthermore, CXCL10 may also recruit additional mast cells to the airways, potentially enabling type 1/type 2 collaboration, since mast cells can also elaborate type 2 cytokines (58). Our data suggest that CS are ineffective against the underlying source of type 1 inflammation in this endotype and raise the concern that some SA patients may be harmed over time by $\mathrm{CS}$, given the increased association of GR with STAT1 promoters on the background of type 1 inflammation. Identifying reliable ways of measuring CXCL10 levels without bronchoscopy may help establish CXCL10 as a biomarker for patients unlikely to respond to CS therapy. Furthermore, our work suggests that interventions to disrupt the IFN- $\gamma /$ CXCL10 pathway or the CXCR3 receptor may alleviate disease symptoms in a subset of severe asthmatics.

\section{Methods}

Human subjects. Data previously obtained from patients enrolled in the Severe Asthma Research Program (SARP) I/II cohorts was used. In brief, nonsmoking subjects between ages 18-60 with asthma from racially/ethnically diverse backgrounds were recruited. All patients had $\mathrm{FEV}_{1}>60 \%$ predicted. Patients meeting the American Thoracic Society (ATS) 2000 definition of SA were classified as severe asthmatics, with the remaining patients classified as mild to moderate asthmatics. Additional nonsmoking patients with out asthma were recruited as healthy controls. Patients underwent regularly scheduled visits that included spirometry, exhaled nitric oxide (FeNO), sputum sample, clinical questionnaires, and medication use questionnaires. All data were previously obtained prior to the start of this project.

$B A L$ samples. Samples used were from patients from SARP I/II had who previously undergone fiberoptic bronchoscopy with BAL using a previously published protocol (31). Recovered fluid was centrifuged to obtain cells, and mRNA was extracted as described below.

Mice. BALBc/ByJ mice were purchased from Jackson Laboratory (catalog 001026). Mice were housed in pathogen-free conditions and underwent treatments between 8-10 weeks of age. Age-matched male mice were used in this study.

Mouse models of SA and MMA. As described previously (5), in the SA model, BALB/c mice were sensitized to $25 \mu \mathrm{g}$ HDM antigen (low-endotoxin, Greer Laboratories, catalog XPB70D3A2.5) and $5 \mu \mathrm{g}$ cyclicdi-GMP (Axxora, catalog BLG-C057-01) delivered i.n. on days 1, 3, and 5. Mice were rested 5 days, then i.n. challenged with 3 challenge sets consisting of 3 consecutive daily challenges with HDM and cyclic-diGMP with 4 days of rest between each set. Each challenge set included $0.5 \mu$ g cyclic-di-GMP with $25 \mu \mathrm{g}$ HDM on day 1 , then $25 \mu \mathrm{g}$ HDM on the following 2 days. Mice were then sacrificed on day 28 , with lungs obtained for histology and whole lung homogenate. In the MMA model, all steps were similar, except cyclic-di-GMP was omitted. For Dex treatment, $4 \mathrm{mg} / \mathrm{kg}$ of Dex (Fresenius Kabi, catalog 402084D) was administered i.p. on day 1 of each challenge set and repeated every 3 days until the mice were sacrificed.

Cell culture. THP-1 cells (ATCC, catalog TIB-202) cells, which were passaged up to 12 times, were cultured in RPMI 1640 (ATCC, catalog 30-2001) supplemented with $0.05 \mathrm{mM} \beta$-mercaptoethanol (Gibco, catalog 21985-023), $20 \mu \mathrm{g} / \mathrm{ml}$ gentamicin (Gibco, catalog 15710-064), and 10\% FBS (Invitrogen, catalog 10082139). Cells were passaged by centrifugation at $450 \mathrm{~g}$ for 7 minutes and split 1:5. Cells were maintained in $10-\mathrm{cm}$ cell culture dishes in an incubator at $37^{\circ} \mathrm{C}$ with $5 \% \mathrm{CO}_{2}$. For experiments, cells were plated in 6-, 12-, or 24-well tissue culture plates (Falcon, catalogs 08-772-1B, 08-772-29, 08-772-1, respectively).

Primary human monocytes. Leukopaks were obtained from the Central Blood Bank Research Products Department and peripheral blood mononuclear cells (PBMCs) were isolated by Ficoll separation (Thermo Fisher Scientific, catalog 45-001-749). Cells were then stored in liquid nitrogen in 1:1 mix of culture medium and DMSO (Sigma-Aldrich, catalog D2650) until use. Cells were thawed and sorted using anti-CD14 magnetic beads for monocyte selection (Miltenyi Biotec, catalog 130-050-201) passing through a preseparation filter (Miltenyi Biotec, catalog 120-007-534) and using a magnetic column (Miltenyi Biotec, catalog 130-042-401). Cells were then collected and plated in 24-well tissue culture plates in X-Vivo 15 cell culture medium (Lonza, catalog 04-418Q). 
Cytokines/exposures. Cells were treated with the following cytokines/additives in different experiments: IFN- $\gamma$ (eBioscience, catalog 14-8319-80) at $100 \mathrm{ng} / \mathrm{ml}$, LPS (Sigma-Aldrich, catalog L8274) at $10 \mathrm{ng} / \mathrm{ml}$, and Dex at $1 \mu \mathrm{M}$ and fluticasone (Sigma-Aldrich, catalog F9428) at $100 \mathrm{nM}$.

$m R N A$ isolation and $c D N A$ preparation. THP-1 cells were isolated by centrifugation and the cell pellet was resuspended in RLT-plus buffer (Qiagen, catalog 1048449) (1:100 $\beta$-mercaptoethanol added). Human monocytes were directly lysed in the cell culture plate with RLT-plus buffer (1:100 $\beta$-mercaptoethanol). For mouse samples, lungs were isolated following sacrifice and dissociated in TRIzol (Invitrogen, catalog 10296-028) using a high-speed homogenizer. mRNA isolation was completed using RNeasy kit (Qiagen, catalogs 74134 or 74136 ) per manufacturer's protocol. cDNA was prepared per manufacturer's protocol using a high-capacity cDNA reverse transcription kit (Thermo Fisher Scientific, catalog 4368813).

Quantitative PCR. qPCR was completed using TaqMan 2x master mix (Thermo Fisher Scientific, catalog 4304437) and validated TaqMan primer/probe sets (Invitrogen) as follows: human HPRT1 (Hs02800695_m1), human CXCL10 (Hs01124251_g1), human DUSP1 (Hs00610256_g1), mouse Hprt (Mm03024075_m1), mouse Ifng (Mm01168134_m1), and mouse Cxcl10 (Mm00445235_m1). SARP BAL mRNA was analyzed with the following primers: CXCL10 (Hs00171042_m1), CPA3 (Hs00157019_m1), IFNG (Hs00989291_m1), tryptase gene (Hs02576518_gH), and GAPDH (4310884E). Results were analyzed with SDS 2.2.2 software, and fold change was calculated with $2^{-\Delta \Delta C t}$ normalized to HPRT/Hprt as internal reference using Excel (Microsoft). For samples without a naive control, gene expression was calculated using $2^{-\Delta \mathrm{Ct}}$ normalized to HPRT1/Hprt as internal reference. SARP samples were calculated with $2^{-\Delta \mathrm{Ct}}$ $\times 1,000$ normalized to $G A P D H$ as internal reference.

ELISA. Supernatants were collected from cell cultures, and CXCL10 protein was measured in clear flat-bottomed 96-well plates (Thermo Fisher Scientific, catalog 14-245-61) by ELISA per manufacturer's protocol (Thermo Fisher Scientific, catalog DY266-05).

Assessment of GR nuclear translocation using imaging cytometry. Staining for cytoplasmic and nuclear GR expression was carried out using fixation and permeabilization buffers contained in the eBioscience FoxP3/transcription factor staining buffer set (Thermo Fisher Scientific, catalog 00-5523-00). Treated cells or untreated controls were fixed for 30 minutes at $4^{\circ} \mathrm{C}$ in permeabilization/fixation buffer, followed by washing with PBS. The cells were resuspended in permeabilization buffer and blocked with $2 \%$ mouse serum for 10 minutes, followed by incubation with purified, unlabeled mouse anti-GR mAb (clone BuGR2; Invitrogen, Thermo Fisher Scientific, catalog MA1-510) for 30 minutes at $4^{\circ} \mathrm{C}(2 \mu \mathrm{g} / 100 \mu 1$ test, according to the manufacturer's recommendations). Following washing with permeabilization buffer, the cells were incubated for 30 minutes at $4^{\circ} \mathrm{C}$ with Alexa Fluor 488-labeled goat anti-mouse IgG cross-adsorbed secondary $\mathrm{Ab}$ (Invitrogen, Thermo Fisher Scientific, catalog A11001) at a 1:500 dilution also in permeabilization buffer. The cells were again washed, resuspended to a concentration of $2 \times 10^{7} \mathrm{cells} / \mathrm{ml}$ in PBS, and placed in $1.5-\mathrm{ml}$ microcentrifuge tubes. Nuclei were counterstained by adding $0.5 \mu 1$ DRAQ5 fluorescent probe solution (Thermo Fisher Scientific, catalog 62251) to each sample 10 minutes prior to data acquisition. Verification of the monocyte population identity was performed by staining with APC-labeled anti-human CD14 mAb (clone M5E2; BD Biosciences, catalog 555399) using the same buffers.

Data acquisition was carried out on stained samples using an ImageStream X Mark II imaging cytometer (Amnis/EMD Millipore) running the manufacturer's Inspire (ISX) software. Brightfield data were collected at 435-480 nm (channel 1), side scatter at 745-780 nm (channel 6), Alexa Fluor 488-labeled GR at 480-560 nm (channel 2), and nuclear staining with DRAQ5 at 640-745 nm (channel 11). A compensation matrix was calculated with single-stained samples using the software's compensation wizard. Cells with an aspect ratio approaching 1 (approximately rounded, single cells) and a comparatively larger surface area (Supplemental Figure 1A, left panel) were confirmed to be monocytes using staining with CD14 (Supplemental Figure 1A, right panel). Lymphocytes, with an identical aspect ratio but smaller overall area, were CD14-negative (Supplemental Figure $1 \mathrm{~A}$, middle panel). Data were collected for $1 \times 10^{4}$ monocytes per sample. Analysis of the raw image files (.rif) generated during acquisition was carried out using Image Data and Analysis Software (IDEAS, Amnis). The gradient RMS feature of IDEAS, which essentially detects variation in pixel intensities on the cell membrane, was first used to ensure that the cells were in focus. The plot of gradient RMS versus normalized frequency demonstrating a sharp peak indicates that, indeed, the majority of the cells were in focus, and the population within the gate labeled "Cells in Focus" was used for further analysis (Supplemental Figure 1B, left panel). Next, monocytes were identified as during data acquisition using a plot of area versus aspect ratio, with confirmation using CD14 staining also as described above (Supplemental Figure 1B, middle panel). Final gating for analysis 
was set on monocytes expressing both GR and DRAQ5, which included the vast majority of cells (Supplemental Figure 1B, right panel). The IDEAS nuclear translocation wizard was then used to determine the proportion of cells in which GR and DRAQ5 are colocalized. The similarity score generated by IDEAS (essentially a measure of colocalized pixels) was plotted against normalized frequency in order to visualize the results. Finally, visual observation of cells with cytoplasmic (lower similarity score) versus nuclear (higher similarity score) (Figure 4A) was used to set the final gate indicative of presence or absence of nuclear translocation.

Reporter assay. THP-1 cells underwent nucleofection using Cell Line Nucleofector Kit V per manufacturer's protocol (Lonza, catalog VCA-1003). Cells were nucleofected with a plasmid containing the luciferase firefly gene linked upstream to a GRE or the appropriate controls. A second plasmid with constitutive expression of Renilla luciferase was used as a control for transfection efficiency (Cignal, catalog CCS-006L). Cells were rested overnight, treated with different agents, and harvested 24 hours later. Luciferase activity was measured using a Dual Luciferase Reporter kit (Promega, catalog E1960) and a luminometer (GloMax, Promega).

Western blot analysis. Cells were lysed using Cell Lysis Buffer (Cell Signaling Technology, catalog 9803) supplemented with Halt protease/phosphatase inhibitor (Thermo Fisher Scientific, catalog 78447) per manufacturer's protocol. After clearing the lysate by centrifugation, total protein was estimated by Bradford assay. Protein (30 $\mu \mathrm{g}$ of total) was resolved on 10\% SDS-PAGE gel, and proteins were then transferred to polyvinylidenediflouride membrane. The blot was then probed with antibodies to phospho-STAT1-S727 (Cell Signaling Technology, catalog 8826) or phospho-STAT1-t707 (Cell Signaling Technology, catalog 9171) or STAT1 (Cell Signaling Technology, catalog 9172S). The blot was then probed with HRP-conjugated secondary Ab and developed using SuperSignal West Femtochemiluminescence substrate (Thermo Fisher Scientific, catalog PI34096).

ChIP assay. ChIP assays were performed using the Pierce Agarose ChIP kit (Thermo Fisher Scientific, catalog 26156) following manufacturer's instructions. Briefly, THP-1 cells were treated with IFN- $\gamma$ or LPS with or without Dex or left untreated and crosslinked using formaldehyde. The cells were harvested by centrifugation, and pellet was resuspended in lysis buffer provided with the kit. Chromatin was immunoprecipitated using antibodies against STAT1 (Cell Signaling Technology, catalog 9172), GR (Thermo Fisher Scientific, catalog MA-1510), or NF-kB (p65) (Abcam, catalog ab7970). Immunoprecipitated genomic DNA was amplified using the following primers: NF-кB1 (FP 5' AAAACCTGCTGGCTGTTCCT3', RP 5' AAAAGAGGAGCAGAGGGAAA 3'); NF-кB 2 (FP 5' AGCCATTTTCCCTCCCTAAT 3', RP 5' CCTCTGAGTCTGCAACATGG 3'); ISRE (FP 5' CATCTTGCAGACTCGAAGGT 3', RP 5' TGGATTGCAACCTTTGTTTT 3'); STAT1 (FP 5' TGTGCAATGGTACCAAGGTG 3', RP 5 GCAAAAGCCTGCCTCAAA 3').

Statistics. Population data for SARP I/II are shown as mean with 25th-75th percentile. Multiple group population data were nonparametric by D'Agostino normality testing and analyzed by Kruskal-Wallis with Dunn's post-test for multiple comparisons. Categorical and binary data were analyzed using Fisher's exact test. Two group comparisons (CXCL10 Low compared with CXCL10 High) were completed using 2-tailed Mann-Whitney $U$ test. Benchtop and mouse studies were analyzed by one-way ANOVA with Tukey's post-test for multiple comparisons for multiple groups and 2-tailed Mann-Whitney $U$ test for 2 groups. Correlations were calculated using Spearman's nonparametric correlation. Matched samples were analyzed using Wilcoxon matched pairs. A $P$ value of less than 0.05 was considered significant. All calculations were completed in Prism 7.0 (GraphPad).

Study Approval. For human samples, written informed consent was obtained from participants prior to inclusion in the study. All samples and data were collected with approval of the IRB for the University of Pittsburgh. All animal-related protocols, housing, and care were approved by the IACUC for the University of Pittsburgh.

\section{Author contributions}

AR and MG conceived the study. MG, KC, TBO, SEW, and AR designed the experiments. SEW performed the human subject bronchoscopies and provided the human BAL cells. MG, KC, TBO, MR, SD, JC, AS, and $\mathrm{RH}$ performed experiments and analyzed the data. AR, MG, SEW, and PR analyzed the data. MF assisted with the mast cell gene expression analysis. MG and AR wrote the manuscript.

\section{Acknowledgments}

The authors thank Donald DeFranco for helpful suggestions. This work was supported by the NIH grants F32HL137089 (to MG); AI106684, HL113956, and AI048927 (to AR); AI100012, HL114453, and HL122307 (to PR); and HL69174 (to SEW). This work benefited from IMAGESTREAMX MARKII funded by NIH 1S10OD019942-01 (Department of Immunology) and T32HL007563 (Divi- 
sion of Pulmonary, Allergy and Critical Care Medicine, Department of Medicine, University of Pittsburgh School of Medicine; Principal Investigator: Rama Mallampalli). We thank S. Nouraie for advice on statistical analysis of data.

Address correspondence to: Anuradha Ray, or Sally E. Wenzel, Department of Medicine, Pulmonary, Allergy and Critical Care Medicine, University of Pittsburgh School of Medicine, 3459 Fifth Avenue, MUH A628 NW Pittsburgh, Pennsylvania 15213, USA. Phone: 412.802.3191; Email: raya@pitt.edu (A. Ray); wenzelse@upmc.edu (S.E. Wenzel).

1. Wenzel SE. Asthma phenotypes: the evolution from clinical to molecular approaches. Nat Med. 2012;18(5):716-725

2. Gauthier M, Ray A, Wenzel SE. Evolving Concepts of Asthma. Am J Respir Crit Care Med. 2015;192(6):660-668.

3. Ray A, Oriss TB, Wenzel SE. Emerging molecular phenotypes of asthma. Am J Physiol Lung Cell Mol Physiol. 2015;308(2):L130-L140.

4. Woodruff PG, et al. T-helper type 2-driven inflammation defines major subphenotypes of asthma. Am J Respir Crit Care Med. 2009;180(5):388-395.

5. Raundhal M, et al. High IFN- $\gamma$ and low SLPI mark severe asthma in mice and humans. J Clin Invest. 2015;125(8):3037-3050.

6. Voraphani N, et al. An airway epithelial iNOS-DUOX2-thyroid peroxidase metabolome drives Th1/Th2 nitrative stress in human severe asthma. Mucosal Immunol. 2014;7(5):1175-1185.

7. Dahlberg PE, Busse WW. Is intrinsic asthma synonymous with infection? Clin Exp Allergy. 2009;39(9):1324-1329.

8. Ray A, Raundhal M, Oriss TB, Ray P, Wenzel SE. Current concepts of severe asthma. J Clin Invest. 2016;126(7):2394-2403.

9. Sallusto F, Lenig D, Mackay CR, Lanzavecchia A. Flexible programs of chemokine receptor expression on human polarized T helper 1 and 2 lymphocytes. J Exp Med. 1998;187(6):875-883.

10. Luster AD, Unkeless JC, Ravetch JV. Gamma-interferon transcriptionally regulates an early-response gene containing homology to platelet proteins. Nature. 1985;315(6021):672-676.

11. Groom JR, Luster AD. CXCR3 ligands: redundant, collaborative and antagonistic functions. Immunol Cell Biol. 2011;89(2):207-215.

12. Tworek D, Kuna P, Młynarski W, Górski P, Pietras T, Antczak A. MIG (CXCL9), IP-10 (CXCL10) and I-TAC (CXCL11) concentrations after nasal allergen challenge in patients with allergic rhinitis. Arch Med Sci. 2013;9(5):849-853.

13. Antonelli A, Ferrari SM, Giuggioli D, Ferrannini E, Ferri C, Fallahi P. Chemokine (C-X-C motif) ligand (CXCL)10 in autoimmune diseases. Autoimmun Rev. 2014;13(3):272-280.

14. Ohmori Y, Hamilton TA. Cooperative interaction between interferon (IFN) stimulus response element and kappa B sequence motifs controls IFN gamma- and lipopolysaccharide-stimulated transcription from the murine IP-10 promoter. J Biol Chem. 1993;268(9):6677-6688.

15. Spurrell JC, Wiehler S, Zaheer RS, Sanders SP, Proud D. Human airway epithelial cells produce IP-10 (CXCL10) in vitro and in vivo upon rhinovirus infection. Am J Physiol Lung Cell Mol Physiol. 2005;289(1):L85-L95.

16. Tamassia N, et al. Molecular mechanisms underlying the synergistic induction of CXCL10 by LPS and IFN-gamma in human neutrophils. Eur J Immunol. 2007;37(9):2627-2634.

17. Brightling CE, et al. The CXCL10/CXCR3 axis mediates human lung mast cell migration to asthmatic airway smooth muscle Am J Respir Crit Care Med. 2005;171(10):1103-1108

18. Ichikawa A, et al. CXCL10-CXCR3 enhances the development of neutrophil-mediated fulminant lung injury of viral and nonviral origin. Am J Respir Crit Care Med. 2013;187(1):65-77.

19. Takaku Y, Nakagome K, Kobayashi T, Hagiwara K, Kanazawa M, Nagata M. IFN- $\gamma$-inducible protein of $10 \mathrm{kDa}$ upregulates the effector functions of eosinophils through $\beta 2$ integrin and CXCR3. Respir Res. 2011;12:138.

20. Bochner BS, Hudson SA, Xiao HQ, Liu MC. Release of both CCR4-active and CXCR3-active chemokines during human allergic pulmonary late-phase reactions. J Allergy Clin Immunol. 2003;112(5):930-934.

21. Miotto D, et al. Expression of IFN-gamma-inducible protein; monocyte chemotactic proteins 1, 3, and 4; and eotaxin in TH1and TH2-mediated lung diseases. J Allergy Clin Immunol. 2001;107(4):664-670.

22. Ying $\mathrm{S}$, et al. Thymic stromal lymphopoietin expression is increased in asthmatic airways and correlates with expression of Th2-attracting chemokines and disease severity. J Immunol. 2005;174(12):8183-8190.

23. Machura E, et al. Selected CC and CXC chemokines in children with atopic asthma. Postepy Dermatol Alergol. 2016;33(2):96-101.

24. Hall DJ, Bates ME, Guar L, Cronan M, Korpi N, Bertics PJ. The role of p38 MAPK in rhinovirus-induced monocyte chemoattractant protein-1 production by monocytic-lineage cells. J Immunol. 2005;174(12):8056-8063.

25. Korpi-Steiner NL, Valkenaar SM, Bates ME, Evans MD, Gern JE, Bertics PJ. Human monocytic cells direct the robust release of CXCL10 by bronchial epithelial cells during rhinovirus infection. Clin Exp Allergy. 2010;40(8):1203-1213.

26. Medoff BD, et al. IFN-gamma-inducible protein 10 (CXCL10) contributes to airway hyperreactivity and airway inflammation in a mouse model of asthma. J Immunol. 2002;168(10):5278-5286.

27. Lin Y, et al. Attenuation of antigen-induced airway hyperresponsiveness and inflammation in CXCR3 knockout mice. Respir Res. 2011;12:123.

28. Sun D, Ding A. MyD88-mediated stabilization of interferon-gamma-induced cytokine and chemokine mRNA. Nat Immunol. 2006;7(4):375-381.

29. Hao S, Baltimore D. The stability of mRNA influences the temporal order of the induction of genes encoding inflammatory molecules. Nat Immunol. 2009;10(3):281-288.

30. Abraham SM, Clark AR. Dual-specificity phosphatase 1: a critical regulator of innate immune responses. Biochem Soc Trans. 2006;34(Pt 6):1018-1023.

31. Balzar S, et al. Mast cell phenotype, location, and activation in severe asthma. Data from the Severe Asthma Research Program. Am J Respir Crit Care Med. 2011;183(3):299-309. 
32. Gordon ED, et al. Alternative splicing of interleukin-33 and type 2 inflammation in asthma. Proc Natl Acad Sci USA 2016;113(31):8765-8770.

33. Chung KF, et al. International ERS/ATS guidelines on definition, evaluation and treatment of severe asthma. Eur Respir J. 2014;43(2):343-373

34. Akinbami LJ, et al. Asthma outcomes: healthcare utilization and costs. J Allergy Clin Immunol. 2012;129(3 Suppl):S49-S64.

35. Barnett SB, Nurmagambetov TA. Costs of asthma in the United States: 2002-2007. J Allergy Clin Immunol. 2011;127(1):145-152.

36. Szefler SJ, et al. Economic burden of impairment in children with severe or difficult-to-treat asthma. Ann Allergy Asthma Immunol. 2011;107(2):110-119.e1.

37. Oriss TB, et al. IRF5 distinguishes severe asthma in humans and drives Th1 phenotype and airway hyperreactivity in mice. JCI Insight. 2017;2(10):91019.

38. Kurashima K, Fujimura M, Myou S, Ishiura Y, Onai N, Matsushima K. Asthma severity is associated with an increase in both blood CXCR3+ and CCR4+ T cells. Respirology. 2006;11(2):152-157.

39. Groom JR, et al. CXCR3 chemokine receptor-ligand interactions in the lymph node optimize CD4+ T helper 1 cell differentiation. Immunity. 2012;37(6):1091-1103.

40. Kohlmeier JE, et al. CXCR3 directs antigen-specific effector CD4+ T cell migration to the lung during parainfluenza virus infection. J Immunol. 2009;183(7):4378-4384.

41. Deiuliis JA, et al. Pulmonary T cell activation in response to chronic particulate air pollution. Am J Physiol Lung Cell Mol Physiol. 2012;302(4):L399-L409.

42. Liu Z, Chen H, Chen X, Gao J, Guo Z. Characteristics of Allergic Pulmonary Inflammation in CXCR3Knockout Mice Sensitized and Challenged with House Dust Mite Protein. PLoS ONE. 2016;11(10):e0162905.

43. Ma B, et al. Role of CCR5 in IFN-gamma-induced and cigarette smoke-induced emphysema. J Clin Invest. 2005;115(12):3460-3472.

44. Korpi-Steiner NL, Bates ME, Lee WM, Hall DJ, Bertics PJ. Human rhinovirus induces robust IP-10 release by monocytic cells, which is independent of viral replication but linked to type I interferon receptor ligation and STAT1 activation. J Leukoc Biol. 2006;80(6):1364-1374.

45. Haché RJ, Tse R, Reich T, Savory JG, Lefebvre YA. Nucleocytoplasmic trafficking of steroid-free glucocorticoid receptor. J Biol Chem. 1999;274(3):1432-1439.

46. Ray A, Prefontaine KE. Physical association and functional antagonism between the p65 subunit of transcription factor NF-kappa B and the glucocorticoid receptor. Proc Natl Acad Sci USA. 1994;91(2):752-756.

47. Aittomäki S, Pesu M, Groner B, Jänne OA, Palvimo JJ, Silvennoinen O. Cooperation among Stat1, glucocorticoid receptor, and PU.1 in transcriptional activation of the high-affinity Fc gamma receptor I in monocytes. J Immunol. 2000;164(11):5689-5697.

48. Sutterwala FS, Noel GJ, Salgame P, Mosser DM. Reversal of proinflammatory responses by ligating the macrophage Fcgamma receptor type I. J Exp Med. 1998;188(1):217-222.

49. Bhandare R, et al. Glucocorticoid receptor interacting protein-1 restores glucocorticoid responsiveness in steroid-resistant airway structural cells. Am J Respir Cell Mol Biol. 2010;42(1):9-15.

50. Hong H, Kohli K, Garabedian MJ, Stallcup MR. GRIP1, a transcriptional coactivator for the AF-2 transactivation domain of steroid, thyroid, retinoid, and vitamin D receptors. Mol Cell Biol. 1997;17(5):2735-2744.

51. Damera G, Tliba O, Panettieri RA. Airway smooth muscle as an immunomodulatory cell. Pulm Pharmacol Ther. 2009;22(5):353-359.

52. Oakley RH, Jewell CM, Yudt MR, Bofetiado DM, Cidlowski JA. The dominant negative activity of the human glucocorticoid receptor beta isoform. Specificity and mechanisms of action. J Biol Chem. 1999;274(39):27857-27866.

53. Wu TR, et al. SHP-2 is a dual-specificity phosphatase involved in Stat1 dephosphorylation at both tyrosine and serine residues in nuclei. J Biol Chem. 2002;277(49):47572-47580.

54. Cahill KN, et al. KIT Inhibition by Imatinib in Patients with Severe Refractory Asthma. N Engl J Med. 2017;376(20):1911-1920.

55. Yu M, et al. Identification of an IFN- $\gamma /$ mast cell axis in a mouse model of chronic asthma. J Clin Invest. 2011;121(8):3133-3143.

56. Barrios VE, Jarosinski MA, Wright CD. Proteinase-activated receptor-2 mediates hyperresponsiveness in isolated guinea pig bronchi. Biochem Pharmacol. 2003;66(3):519-525.

57. O'Connell D, et al. IFN- $\gamma$-induced JAK/STAT, but not NF- $\mathrm{BB}$, signaling pathway is insensitive to glucocorticoid in airway epithelial cells. Am J Physiol Lung Cell Mol Physiol. 2015;309(4):L348-L359.

58. Modena BD, et al. Gene Expression Correlated with Severe Asthma Characteristics Reveals Heterogeneous Mechanisms of Severe Disease. Am J Respir Crit Care Med. 2017;195(11):1449-1463. 